\title{
Physical Processes Related to Discharges in Planetary Atmospheres
}

\author{
R. Roussel-Dupré · J.J. Colman · E. Symbalisty • \\ D. Sentman · V.P. Pasko
}

Received: 19 January 2008 / Accepted: 19 May 2008 / Published online: 18 June 2008

(C) Springer Science+Business Media B.V. 2008

\begin{abstract}
This paper focuses on the rudimentary principles of discharge physics. The kinetic theory of electron transport in gases relevant to planetary atmospheres is examined and results of detailed Boltzmann kinetic calculations are presented for a range of applied electric fields. Comparisons against experimental swarm data are made. Both conventional breakdown and runaway breakdown are covered in detail. The phenomena of transient luminous events (TLEs), particularly sprites, and terrestrial gamma-ray flashes (TGFs) are discussed briefly as examples of discharges that occur in the terrestrial environment. The observations of terrestrial lightning that exist across the electromagnetic spectrum and presented throughout this volume fit well with the broader understanding of discharge physics that we present in this paper. We hope that this material provides the foundation on which explorations in search of discharge processes on other planets can be based and previous evidence confirmed or refuted.
\end{abstract}

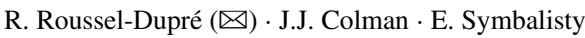

Earth and Environment Sciences Devision, Atmospheric, Climate, and Environmental Dynamics Group, MS F665, Los Alamos National Lab, Los Alamos, NM 87545, USA

e-mail: bobrdnm@comcast.net

J.J. Colman

e-mail: jonah@lanl.gov

E. Symbalisty

e-mail: esymbalisty@lanl.gov

D. Sentman

Physics Department, University of Alaska Fairbanks, 108 Natural Sciences Facility, 708E Elvey Building, Fairbanks, AK 99775, USA

e-mail: dsentman@gi.alaska.edu

V.P. Pasko

Department of Electrical Engineering, Communications and Space Sciences Laboratory (CSSL), The Pennsylvania State University, 211B Electrical Engineering East, University Park,

PA 16802-2706, USA

e-mail: vpasko@psu.edu 
Keywords Planetary atmospheres · Electrical discharges · Electrical breakdown ·

Lightning · Kinetic theory · Swarm · Electron transport · Atmospheric electricity ·

Boltzmann equation - Fokker-Planck equation - Relativistic breakdown · Ionization · Drift velocity $\cdot$ Characteristic energy $\cdot$ Electron impact cross-sections $\cdot$ Electron attachment

\section{Electron Transport and Avalanche in Gases}

The acceleration, scattering, and energy loss or gain experienced by an electron as it moves through a gas subject to an applied electric field depends entirely on the gas composition, the details of the electron interactions with the constituent particles, and the boundary conditions. For weak fields the electrons drift and diffuse through the gas while undergoing elastic and inelastic collisions that together with the field define their momentum and energy distribution. The inelastic interactions that can occur include rotational, vibrational, and electronic excitations of the gas particles as well as losses by way of attachment and recombination. For stronger fields it is possible for ionizing collisions to take place. A gas discharge is initiated when the applied electric field exceeds the threshold value necessary for a sufficient population of electrons to overcome collisional drag and accelerate to energies beyond the gas ionization potential. In addition the ionization rate must exceed the net dissociative attachment rate (if extant) in order to have a net growth in the electron population. The energy or electric field at which the two balance each other defines the threshold for a discharge to initiate. Three-body attachment may also play an essential role in defining the overall development of the discharge as in air.

To date, two electrical breakdown mechanisms are known to operate in dielectrics. The first is the conventional breakdown (CB) process that has been studied extensively in the laboratory for a century or more and that is recognized as the sparks, arcs, and glow discharges of routine occurrence (cf., Loeb 1939; Raether 1964; Raizer 1991; Bazelyan and Raizer 1998). The second is a relatively new mechanism called runaway breakdown (RB) that was first advanced by Gurevich et al. (1992) and involves an avalanche of relativistic electrons that are collimated by the applied field to form an electron beam. RB may play an important role in lightning discharges on Earth (cf., Gurevich and Zybin 2005). Many of the fundamental ideas associated with electron runaway in thunderstorm electric fields were discussed by Wilson $(1925,1956)$.

Both breakdown mechanisms can be understood in the context of Fig. 1 where the frictional force, normalized to the minimum value at high energies, experienced by an electron moving through air is plotted as a function of the electron energy. This plot was derived by calculating the electron energy loss per unit length due to translational, rotational, vibrational, electronic, and ionizing collisions with air molecules. At high energies beyond $\sim 10 \mathrm{keV}$ the plotted values agree well with the Bethe energy loss expression (Bethe 1930; Bethe and Ashkin 1953) which is often referred to as the dynamical friction force. We see that a local minimum corresponding to $218 \mathrm{keV} / \mathrm{m}$ in sea level air exists at approximately 1.4 MeV. Clearly, if an electric field whose magnitude exceeds the minimum is applied to the medium then electrons with energies greater than the critical value $\varepsilon_{c}$ at which the electric force equals the frictional force (see Fig. 1 for the case of an applied field equal to the conventional breakdown field where $\varepsilon_{c}$ is approximately $10 \mathrm{keV}$ ) will be maintained and accelerated (runaway) to higher energies. It is also true that impact ionization of the air by energetic electrons will lead to the production of energetic secondary electrons. Those secondary electrons whose energies exceed the critical value $\varepsilon_{c}$ become part of the runaway population and contribute to further ionization that also populates the runaway regime. The 


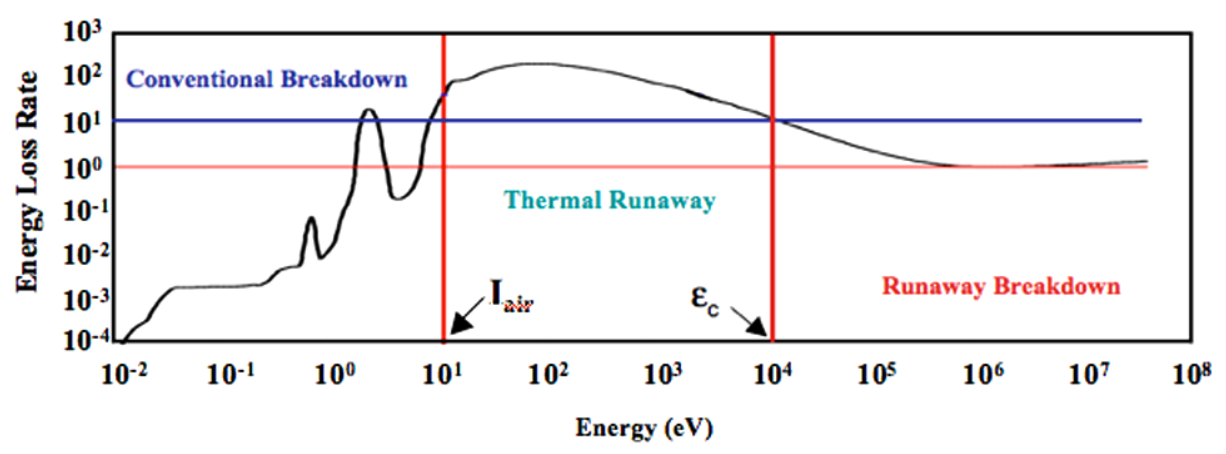

Fig. 1 Rough sketch of the electron energy loss rate in air, normalized to the local minimum at approximately 1.4 $\mathrm{MeV}$, as a function of electron energy. The blue line represents the threshold for conventional breakdown which is ten times the threshold for runaway (shown in red). The critical energy $\varepsilon_{c}$ at which electrons runaway in the presence of the conventional breakdown threshold field is shown at $\sim 10 \mathrm{keV}$. The ionization potential for air $(\sim 14.9 \mathrm{eV})$ is also shown

net result is an avalanche in which the electron population grows exponentially. Collimation of these relativistic electrons by the electric field leads to the formation of an electron beam that grows exponentially as it propagates through the medium as long as the electric field exceeds a threshold defined near the minimum of the frictional force. In general, RB initiation requires a seed energetic electron with energy of order 1-10s of keV depending on the field strength. In planetary atmospheres, at altitudes below the ionosphere or the layer where solar UV and EUV radiation is absorbed, cosmic ray (CR) interactions provide the necessary seed population for initiation.

In the case of $\mathrm{CB}$ an applied electric field accelerates seed thermal electrons $(\sim 0.03 \mathrm{eV}$ at STP) against the frictional drag such that some fraction of the electrons reach or exceed the ionization potential of air and eject additional secondary electrons that accelerate to sufficient energies to produce additional tertiary electrons and so on. The ensuing avalanche is limited in its energy extent by the large and broad maximum in the ionization energy loss rate depending on the field strength. Near the threshold for $\mathrm{CB}$ the electron distribution function is characterized by a mean energy of a few $\mathrm{eV}$ (controlled by the nitrogen resonant vibrational peak near $2 \mathrm{eV}$ ) together with a high-energy tail (tens of eV) that drives the avalanche. When the electric force exceeds the maximum energy loss rate due to ionization then a process referred to as cold or thermal runaway develops and feeds the RB mechanism. In fully and partially ionized gases (Dreicer 1959, 1960) the threshold is known as the Dreicer field which was derived using the Fokker-Planck treatment for electron transport described below. The question of whether or not such high fields $(\sim 10$ times the CB threshold) can be established in the natural environment remains an ongoing topic of debate. This mechanism would compete with cosmic rays as seeds for the runaway process.

One important feature of $\mathrm{RB}$ is that the threshold electric field needed to initiate the avalanche is a factor of ten below that for conventional breakdown (see Fig. 1). Interestingly, macroscopic field strengths near or exceeding the threshold for CB have never been measured in terrestrial thunderstorms while values near and exceeding the threshold for RB have often been measured (see e.g., Marshall et al. 2005; Stolzenburg et al. 2007). At the same time it is important to note that positive and negative leaders can propagate in long gaps with sizes exceeding several tens of meters at ground pressure (Raizer 1991, p. 362) at fields that are significantly below the CB threshold but the question of how the leader is initiated in the first place particularly for lightning remains unanswered (e.g., Uman 2001, p. 79; Raizer 
1991, p. 370; Bazelyan and Raizer 1998, pp. 203, 253). One of the unique signatures of runaway breakdown is the strong $\gamma$-ray flux produced by the beam interaction with the gas. The past several decades of research into the phenomenon of terrestrial lightning has in fact seen an accumulation of evidence for the existence of penetrating radiation (X-and $\gamma$-rays) in direct association with many forms of the lightning discharge (McCarthy and Parks 1985; McCarthy and Parks 1992; Fishman et al. 1994; Eack et al. 1996; Moore et al. 2001; Dwyer 2003; Smith et al. 2005). At high altitudes above $25 \mathrm{~km}$, the Earth's atmosphere becomes transparent to the gamma rays produced by RB and remote detection becomes feasible as in the case of the terrestrial gamma ray flashes (TGFs) measured by the BATSE and RHESSI satellite based detectors (Fishman et al. 1994; Smith et al. 2005; Østgaard et al. 2008; Grefenstette et al. 2008).

While the basic properties of the electron beam formed in runaway breakdown such as the full electron energy distribution function (Symbalisty et al. 1998; Babich et al. 2001), the physical dimensions (Roussel-Dupré and Gurevich 1996; Babich et al. 2008), the diffusion coefficients (Gurevich et al. 1994), and the avalanche rates (Symbalisty et al. 1998; Lehtinen et al. 1999; Babich et al. 2001; and Dwyer 2003) have been studied with detailed kinetic calculations and some initial laboratory experiments have been performed (Gurevich et al. 1999; Babich et al. 2002), precise and comprehensive experimental validation is not presently available. Recently a new source of high-energy electrons in the Earth's magnetosphere due to Compton scattering and pair production by TGFs near the tropopause has been identified (Dwyer et al. 2008). Because of the large avalanche scale lengths (of order tens of meters at atmospheric pressure) necessary to produce an observable effect, RB is not easily reproduced in the laboratory. As a result, the natural environment provides the primary means for studying the details of this mechanism and satellite missions directed towards Earth and other planets provide important platforms for fielding critical diagnostics.

The RB mechanism can be suppressed by an applied magnetic field when the energy dependent electron gyro-frequency $(=e B / \gamma m c$, where $B$ is the magnetic field strength and $\gamma$ is the Lorentz factor) becomes comparable to the electron scattering rate (cf., Gurevich et al. 1996; Lehtinen et al. 1999). This condition is met for the relativistic electrons above approximately $30-40 \mathrm{~km}$ altitude in the terrestrial atmosphere. Once the electron becomes magnetized it follows the field lines. In this way the geomagnetic field acts as an energy filter as a function of height. The pitch angle distribution of the electrons once magnetized will span a broad angular range that depends on the angle of the magnetic field relative to the driving electric field and the runaway distribution function itself in the region above the thunderstorm. Another important effect is that the total current generated in an RB discharge can be significant (tens of kA) and can lead to a self magnetic field approaching and exceeding the geomagnetic field. This beamed plasma is subject to various forms of plasma instabilities that can affect the development of RB and further broaden electron pitch-angle distributions. This RB regime has yet to be explored and may be accessed in TLEs and TGFs.

\section{Kinetic Theory}

The kinetic theory of non-uniform gases was put on a firm mathematical and statistical foundation by Maxwell and Boltzmann by the end of the 19th century. The kinetic treatment of electron transport in gases that was also formulated nearly a century ago (see discussion in Chapman and Cowling 1970) forms the basis even today for calculating the momentum distribution and the statistical motion of electrons through a gas subjected to an applied 
electric field. Cross-sections that quantify the probability of elastic or inelastic collisions are an important ingredient in the kinetic formulation and can also be derived from the interaction potentials for specific processes (e.g., rotational, vibrational, electronic, or ionizing interactions) or measured in the laboratory.

The Boltzmann equation in its most general form is a six dimensional integro-differential equation. The frequently referenced and utilized Maxwell-Boltzmann distribution function is a solution of this equation and it describes the probability distribution of particle momentum and energy for a uniform gas in thermodynamic equilibrium. The Boltzmann equation is still used even today to study the kinetic properties of gases and how they evolve in time. Much of the progress made in our understanding since the seminal works of Maxwell and Boltzmann is based on approximations that make the Boltzmann equation mathematically more tractable. In particular the assumption that the mean-free-path between binary collisions is small compared to the scale lengths that characterize non-uniformities in gases forms the basis for an expansion about the uniform state. Chapman and Enskog employed this approximation to develop a rigorous mathematical formulation that yielded the transport coefficients of particle diffusion, viscosity, the stress tensor, electrical conduction, heat conduction, and thermal diffusion. Inherent to this analysis is the assumed form of the interaction potential that characterizes the outcome of collisions between two particles. Simple models that ranged from rigid elastic spheres to spherically symmetric fields of force that fall off as a specified power of the distance from the particle center were utilized.

These overly simplified forms for the interaction potentials proved inadequate as more and more detail showing the complexity of molecular structures was gathered by spectroscopic means. Eventually, these problems were circumvented in part by relying on measurements of cross-sections that describe the probability of a certain outcome (scattering, momentum transfer, and/or energy loss) following a collision. In the modern computing era in fact it is possible to solve the Boltzmann equation numerically without resorting to analytic formulations and other simplifications. This approach is outlined below in our treatment of electrical discharges. With a full accounting of the relevant cross-sections as a function of the electron energy and scattering angle it is possible to compute the electron velocity distribution function from either the Boltzmann equation or from statistical Monte Carlo calculations that simulate many electron encounters. The Boltzmann equation when solved numerically can be effected by significant numerical diffusion depending on the details of the grid chosen to represent the momentum and spatial domains. The accuracy of Monte Carlo calculations on the other hand depends strongly on the number of particles and trajectories chosen per simulation and the extent of the spatial and momentum volumes. Generally, the Boltzmann formulation has an advantage at high gas densities (large numbers of collisions) and vice versa. However, the two methods have been checked against each other in the case of RB with discrepancies in the avalanche rates of only a few to tens of percent (Babich et al. 2001). Good agreement between discharge characteristics obtained by both methods has also been demonstrated in the conventional breakdown regime (e.g., Moss et al. 2006, and references therein). Our discussions below will focus on the Boltzmann treatment of electron transport.

\subsection{Kinetic Equations for Runaway Breakdown}

Assuming a spatially uniform applied electric field (no spatial dependence) we need only consider two momentum coordinates (azimuthal symmetry exists about the electric field direction) which in a spherical geometry are the momentum amplitude $p$ and the cosine of 
the angle between the applied electric field and the electron momentum direction $\mu$. In this coordinate system the relativistic Boltzmann equation can be written:

$$
\frac{\partial f}{\partial t}-\left[\frac{\left(1-\mu^{2}\right)}{p} \frac{\partial f}{\partial \mu}+\mu \frac{\partial f}{\partial p}\right] e E=\frac{\partial_{e} f}{\partial t}
$$

where $f(t, p, \mu)$ is the electron distribution function, $m$ is the electron mass, $E$ is a self consistent or applied electric field, and $\partial_{e} f / \partial t$ is the Boltzmann collision integral for electrongas interactions. Electron-gas interactions are, at high energies, primarily Coulomb in nature and the electrons undergo small deflections in both velocity space and configuration space. In this limit, the collision integral reduces to a Fokker-Planck form (derivation provided in Chapman and Cowling 1970; Krall and Trivelpiece 1973) that is given by:

$$
\frac{\partial_{e} f}{\partial t}=\frac{1}{p^{2}} \frac{\partial\left(p^{2} F_{D} f\right)}{\partial p}+\frac{(1+Z / 2) F_{D}}{4 \gamma p} \frac{\partial}{\partial \mu}\left[\left(1-\mu^{2}\right) \frac{\partial f}{\partial \mu}\right]+Q_{i o n}
$$

where $Z$ is the mean molecular charge, $\gamma$ is the Lorentz factor $\left(=1 / \sqrt{1-\beta^{2}}, \beta=v / c\right.$, $v=$ electron speed, $c=$ speed of light), $F_{D}$ is the dynamical friction force first obtained by Bethe, namely,

$$
\begin{aligned}
F_{D}= & \frac{4 \pi Z e^{4} N_{m}}{m c^{2}} \frac{\gamma^{2}}{\gamma^{2}-1}\left[\ln \left(\frac{m c^{2} \sqrt{\left(\gamma^{2}-1\right)(\gamma-1)}}{I \sqrt{2}}\right)\right. \\
& \left.+\left(\frac{2}{\gamma}-\frac{1}{\gamma^{2}}\right) \frac{\ln 2}{2}+\frac{1}{2 \gamma^{2}}+\frac{(\gamma-1)^{2}}{16 \gamma^{2}}\right]
\end{aligned}
$$

with $I$ equal to the mean excitation potential of the gas, $e$ is the electron charge, $N_{m}$ is the molecular gas density, and $Q_{i o n}$ is an ionization term that accounts for the production of secondary energetic electrons by more energetic primary electrons. This term is essential for modeling the effects of runaway breakdown (e.g. Gurevich et al. 1992, 1996, 1998; Roussel-Dupré et al. 1994; Roussel-Dupré and Gurevich 1996) and is given by,

$$
Q_{i o n}=N_{m} c \frac{\beta}{\gamma^{2}-1} \frac{2 \pi Z e^{4}}{m c^{2}} \int_{\varepsilon_{L}}^{\infty} d \varepsilon^{\prime} \gamma^{\prime 2} Q_{M}\left(\varepsilon, \varepsilon^{\prime}\right) \frac{1}{\pi} \int_{0}^{\pi} d \alpha f\left(t, \varepsilon^{\prime}, \mu^{\prime}\right)
$$

where $\varepsilon$ is the electron kinetic energy, and the Møller cross section $Q_{M}$ is given by,

$$
Q_{M}\left(\varepsilon, \varepsilon^{\prime}\right)=\left[\frac{1}{\varepsilon^{2}}-\frac{1}{\varepsilon\left(\varepsilon^{\prime}-\varepsilon\right)} \frac{\left(2 \varepsilon^{\prime}+m c^{2}\right) m c^{2}}{\left(\varepsilon^{\prime}+m c^{2}\right)}+\frac{1}{\left(\varepsilon^{\prime}-\varepsilon\right)^{2}}+\frac{1}{\left(\varepsilon^{\prime}+m c^{2}\right)^{2}}\right]
$$

Experimental results are in agreement with the Møller formula to within $8 \%$ (see e.g. Scott et al. 1951). Equation (2.5) is valid for $\varepsilon>\varepsilon^{\prime}$, and the integration is over all incident electrons with energy $\varepsilon^{\prime}$ greater than $\varepsilon_{L}(=2 \varepsilon)$, and with

$$
\mu^{\prime}=\mu \mu_{0}+\sqrt{\left(1-\mu_{0}^{2}\right)\left(1-\mu^{2}\right)} \cos \alpha
$$

where $\mu_{0}=\sqrt{\frac{\varepsilon\left(\varepsilon^{\prime}+2 m c^{2}\right)}{\varepsilon^{\prime}\left(\varepsilon+2 m c^{2}\right)}}$ is the cosine of the angle between the primary $\mu^{\prime}$ and secondary $\mu$ electron momentum vectors and $\alpha$ is the azimuthal angle around the primary electron's momentum vector. 


\subsection{Kinetic Equations for Conventional Breakdown}

Under the same approximations of a uniform applied electric field and azimuthal symmetry around the field, the non-relativistic Boltzmann equation for the electron distribution function, $f(v, \mu, t)$, can be written in a spherical coordinate system as:

$$
\frac{\partial f}{\partial t}-\left[\frac{\left(1-\mu^{2}\right)}{v} \frac{\partial f}{\partial \mu}+\mu \frac{\partial f}{\partial v}\right] \frac{e E}{m}=\frac{\partial_{e} f}{\partial t}
$$

where $t$ represents time, $e$ the charge on an electron, $m$ the electron mass, and $E$ the applied field. The right hand side of this equation defines changes to the distribution function due to encounters with neutral gas molecules (the collision operator) and can be broken down as:

$$
\begin{aligned}
\frac{\partial_{e} f_{s}}{\partial t}= & N_{m} v \int_{o}^{2 \pi} d \alpha \int_{o}^{\pi} \sin \beta d \beta \sigma_{e}(\beta, v)\left[f_{s}\left(v, \mu^{\prime}\right)-f_{s}(v, \mu)\right] \\
& -N_{m} \frac{m}{M} \frac{1}{v^{2}} \frac{\partial}{\partial v}\left\{\int_{o}^{2 \pi} d \alpha \int_{o}^{\pi} \sin \beta d \beta(1-\cos \beta) \sigma_{e}(\beta, v) v^{4} f_{s}\left(v, \mu^{\prime}\right)\right\} \\
& +\sum_{h} N_{h} v \int_{o}^{2 \pi} d \alpha \int_{o}^{\pi} \sin \beta d \beta\left[f_{s}\left(w, \mu^{\prime}\right) \frac{\mathrm{w}^{2}}{\mathrm{v}^{2}} \sigma_{h}(\beta, w)-f_{s}(v, \mu) \sigma_{h}(\beta, v)\right] \\
& +\sum_{I} N_{I} v \int_{o}^{2 \pi} d \alpha \int_{o}^{\pi} \sin \beta d \beta\left[\int_{0}^{\infty} d \varepsilon_{I} f_{s}\left(\varepsilon_{I}, \mu^{\prime}\right) \frac{\varepsilon_{\mathrm{I}}}{v^{2}} \sigma_{I}\left(\varepsilon_{I}, \varepsilon_{b}\right)-f_{s}(v, \mu) \sigma_{I t o t}(v)\right]
\end{aligned}
$$

where

$$
w^{2}=v_{h}^{2}+v^{2}, \quad \varepsilon_{I}=v_{I}^{2}+v^{2}+\varepsilon_{\mathrm{b}}, \quad \text { and } \quad \mu^{\prime}=\mu \mu_{\beta}+\sqrt{1-\mu^{2}} \sqrt{1-\mu_{\beta}^{2}} \cos \alpha .
$$

Sums are taken over all inelastic states $h$ and ionization states $I . N_{m}$ is the molecular gas density; $\sigma_{e}$ the elastic differential cross-section (angular); $\sigma_{h}$ the inelastic differential crosssection (angular); $\sigma_{I}$ the ionization differential cross-section (energy); $\sigma_{\text {Itot }}$ the total ionization cross-section; $\varepsilon_{I}$ the incident electron energy in ionizing collisions; $\varepsilon_{b}$ the scattered electron energy in ionizing collisions; $v_{h}$ the inelastic threshold velocity for excitation; $v_{I}$ the threshold velocity for ionization; $v$ the electron velocity; and $M$ the molecular mass.

\section{Interaction Cross-Sections for Planetary Gases}

\subsection{Input to Non-relativistic Boltzmann Collision Integral}

In order to solve the Boltzmann equation for electrons a collision integral $\left(\partial_{e} f / \partial t\right)$ must be formulated that represents the interactions between the electrons and the medium through which they travel. At high electron energies the Fokker-Planck form may be used, as the interactions are primarily Coulomb in nature and small angle scattering dominates. At lower electron energies the interactions begin to couple to internal states of the medium, e.g. an electronic excitation of molecular nitrogen, and collisions characterized by large angle scattering and/or energy loss become important. In this case it is necessary to have knowledge of the various internal states of the medium, as well as its susceptibility to ionization and 
attachment, in order to properly formulate the collision integral. Here we will quickly review the current state of knowledge of the cross sections for elastic, inelastic (rotational, vibrational, and electronic), attachment (both three body and dissociative), and ionizing interactions between electrons and those gases relevant to planetary atmospheres: molecular nitrogen $\left(\mathrm{N}_{2}\right)$, molecular oxygen $\left(\mathrm{O}_{2}\right)$, molecular hydrogen $\left(\mathrm{H}_{2}\right)$, carbon dioxide $\left(\mathrm{CO}_{2}\right)$, methane $\left(\mathrm{CH}_{4}\right)$, and helium $(\mathrm{He})$.

We have taken data from the peer-reviewed and institutional literature at times, but there are two additional sources of relevant cross sections available on the Internet. The first is the well-known 'public private communication' by A.V. Phelps, available by ftp at ftp://jila.colorado.edu/collision_data (availability checked as of January 11, 2008); the second is the set of cross sections employed by the Magboltz code maintained by CERN and copyrighted by Biagi (1999). Plots of the Magboltz cross sections are available at http://rjd.web.cern.ch/rjd/cgi-bin/cross and the tabular data can be extracted from the code itself which is available at http://consult.cern.ch/writeups/magboltz/magboltz-7.1.f (availability checked as of January 11, 2008). Both of these sources have references to some of their source publications, and both include modifications or interpretations of their primary sources. The Phelps compilation does not include the elastic cross section for any of the gasses, providing instead an effective momentum transfer cross section, which is appropriate for use in the two-term spherical harmonic expansion treatment. The effective momentum transfer cross section is equal to the sum of the (angular integrated) inelastic cross sections plus the elastic momentum transfer cross section (Pitchford and Phelps 1982). We are not concerned with any form of the momentum transfer cross section here.

It is not our intent to attempt a comprehensive review of the literature here. The most recent 'best value' compilations owe much to earlier work and references can be found in papers referred to here. Our intent is to inform our readers of where readily available tabulated data resides, and give references to the most recent publications that include tabulated data. We will mention two early works in this introduction, both of which were helpful to the authors: the text "The diffusion and drift of electrons in Gases" by Huxley and Crompton (1974), and the review "Electron Scattering by Molecules II. Experimental Methods and Data" by Trajmar et al. (1983). A good deal of additional recent information is available in a review article by Brunger and Buckman (2002), which helps outline the uncertainties in the cross sections but does not recommend specific values.

\subsubsection{Nitrogen}

Both the Phelps and Magboltz compilations cover $\mathrm{N}_{2}$, and in fact the Magboltz compilation references Phelps as its source. Recently Itikawa (2006) published a compilation of his recommended values of the $\mathrm{N}_{2}$ cross-sections; here we focus on those three sources. The various compilations are in reasonable agreement but there are some differences as well. Table 1 lists the various inelastic interactions along with their associated energy loss terms.

In Table 1 we see that Itikawa only specifically recommends values for the $v=0 \rightarrow 1$ transition, he does discuss and provide references to the higher transitions all the way up to $v=0 \rightarrow 17$. The vibrational cross sections utilized by Magboltz are identical to the Phelps compilation. For the electronic transitions the Magboltz treatment starts from work published by Phelps and Pitchford (1985) (JILA Report No. 26), but combines some transitions that are close together in energy. Individual electronic states sometimes show large differences with Itikawa recommending a peak value of $4.73 \times 10^{-17} \mathrm{~cm}^{2}$ for the $a^{\prime 1} \Pi_{g}$ transition at $16.5 \mathrm{eV}$ while Phelps recommends a peak value of $2.023 \times 10^{-17} \mathrm{~cm}^{2}$ at $17 \mathrm{eV}$. Some of these differences are compensated for with respect to the total scattering cross section by differences in other cross sections. 
Table 1 Inelastic processes excited by electron impact with $\mathrm{N}_{2}$ and the associated energy loss

\begin{tabular}{lll}
\hline Excited State & Energy Loss $(\mathrm{eV})$ & Reference \\
\hline Rotation & $0.02,0.00148$ & Phelps, Itikawa \\
Vibrational $(v=0 \rightarrow 1)$ & 0.29 & Phelps, Itikawa \\
Vibrational $(v=0 \rightarrow 2)$ & 0.59 & Phelps, Magboltz \\
Vibrational $(v=0 \rightarrow 3)$ & 0.88 & Phelps, Magboltz \\
Vibrational $(v=0 \rightarrow 4)$ & 1.17 & Phelps, Magboltz \\
Vibrational $(v=0 \rightarrow 5)$ & 1.47 & Phelps, Magboltz \\
Vibrational $(v=0 \rightarrow 6)$ & 1.76 & Phelps, Magboltz \\
Vibrational $(v=0 \rightarrow 7)$ & 2.06 & Phelps, Magboltz \\
Vibrational $(v=0 \rightarrow 8)$ & 2.35 & Phelps, Magboltz \\
$A^{3} \Sigma_{u}^{+}$, Triplet1 & $6.17,6.169,6.17$ & Phelps, Itikawa, Magboltz \\
$B^{3} \Pi_{g}$, Triplet3 & $7.35,7.353,7.35$ & Phelps, Itikawa, Magboltz \\
$W^{3} \Delta_{u}$ & $7.36,7.362$ & Phelps, Itikawa \\
Triplet5 & 7.80 & Magboltz \\
$B^{\prime 3} \Sigma_{u}^{-}$ & $8.16,8.165$ & Phelps, Itikawa \\
$a^{\prime 1} \Sigma_{u}^{-}$ & $8.40,8.399$ & Phelps, Itikawa \\
$a^{1} \Pi_{g}$, Singlet2 & $8.55,8.549$ & Phelps, Itikawa, Magboltz \\
$W^{1} \Delta_{u}$ & $8.89,8.890$ & Phelps, Itikawa \\
$C^{3} \Pi_{u}$, Triplet7 & $11.03,11.032,11.03$ & Phelps, Itikawa, Magboltz \\
$E^{3} \Sigma_{g}^{+}$, Triplet8 & $11.88,11.875,11.87$ & Phelps, Itikawa, Magboltz \\
$a^{\prime \prime 1} \Sigma_{g}^{+}$ & $12.25,12.255$ & Phelps, Itikawa \\
$b^{1} \Pi_{u}$ & 12.500 & Itikawa \\
$c_{4}^{\prime 1} \Sigma_{u}^{+}$ & 12.935 & Itikawa \\
$b^{\prime 1} \Sigma_{u}^{+}$ & 12.854 & Itikawa \\
Sum of singlet, Singlet5 & $13.0,13.0$ & Phelps, Magboltz \\
\hline & & \\
\hline
\end{tabular}

Figure 2a shows a plot of the various $\mathrm{N}_{2}$ cross-sections represented as energy loss per distance traveled at sea level as a function of energy. To construct this plot we have taken the elastic cross section from Magboltz, the inelastic cross sections from Phelps, and the ionization cross section from Itikawa. Figure 2 a also shows the relativistic energy loss calculated from the Bethe formula (2.3).

\subsubsection{Oxygen}

Both the Phelps and Magboltz compilations cover $\mathrm{O}_{2}$. Jeon (2003) has also published a full set of cross sections for $\mathrm{O}_{2}$, which started from the set compiled by Phelps along with higher energy electronic states from a personal communication with M. Hayashi, and adjusted to match experimentally determined transport coefficients calculated from a two-term approximation of the Boltzmann equation. Magboltz again starts with the Phelps compilation but has done more extensive adjustments to match more recent experimental work, including work by Jeon and Nakamura (1998). The various compilations are in reasonable agreement with differences of less than $30 \%$ in most cases. Table 2 lists the various inelastic interactions along with their associated energy loss terms. Figure $2 \mathrm{~b}$ shows a plot of the various $\mathrm{O}_{2}$ cross-sections represented as energy loss per distance traveled at sea level as a function of energy. To construct this plot we have taken the cross sections from the Magboltz compila- 

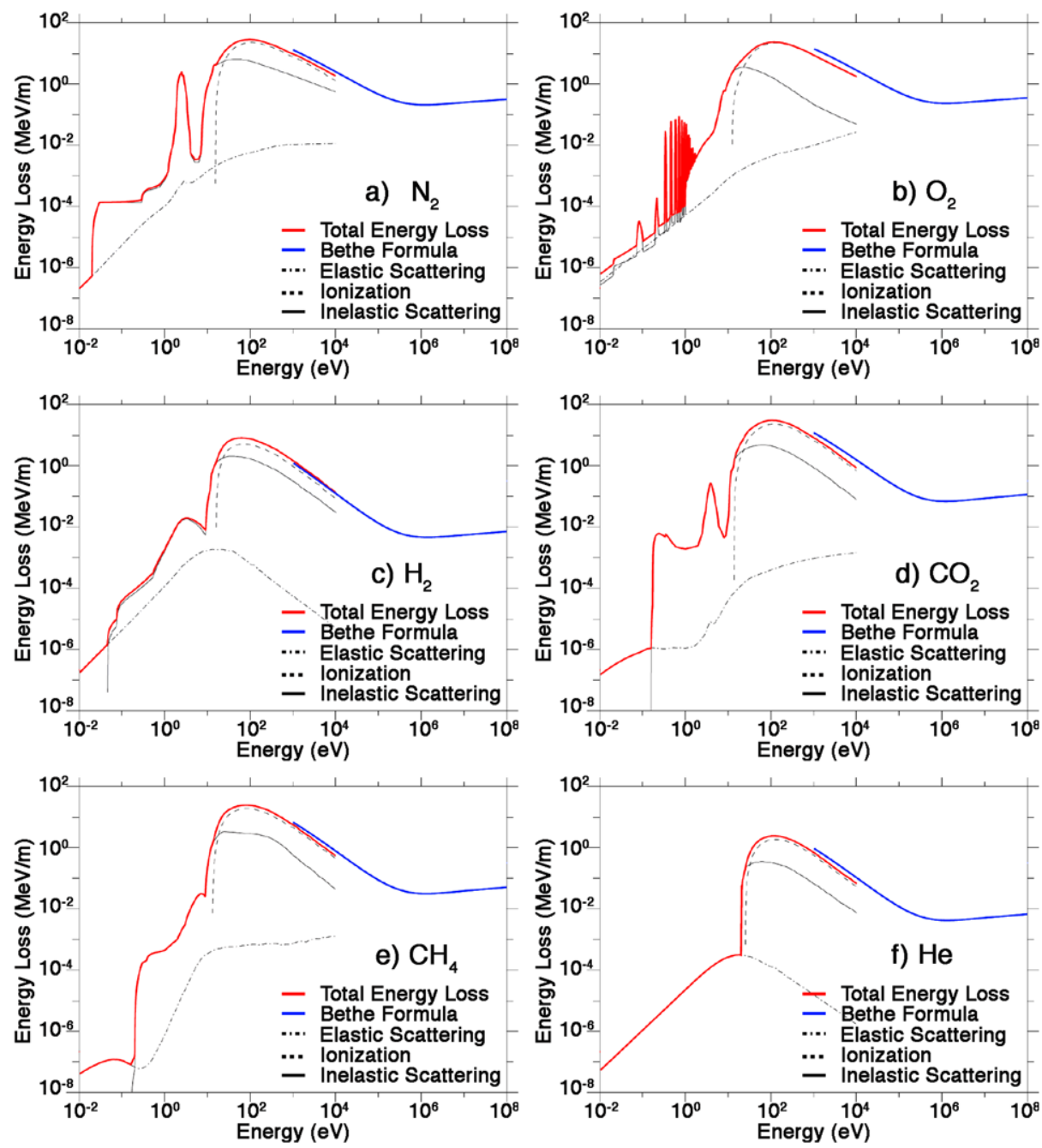

Fig. 2 a-f Energy loss in $\mathrm{MeV} / \mathrm{m}$ for an electron traveling through pure gasses at sea level plotted as a function of electron energy. The total energy loss is represented by the thick red line, elastic scattering by the dash dot pattern, ionization by the dash pattern, and inelastic processes by the thin black line. The blue line was calculated from the Bethe formula (2.3). Figures a-f represent the gases $\mathrm{N}_{2}, \mathrm{O}_{2}, \mathrm{H}_{2}, \mathrm{CO}_{2}, \mathrm{CH}_{4}$, and $\mathrm{He}$ respectively

tion along with an elastic cross section taken from Kanik et al. (1993). It is worth noting that there is large variation among the various published elastic scattering cross section determinations and recommendations. Figure $2 \mathrm{~b}$ also shows the relativistic energy loss calculated from the Bethe formula (2.3).

\subsubsection{Hydrogen}

Both the Phelps and Magboltz compilations cover $\mathrm{H}_{2}$. There is also a useful review by Tawara et al. (1990a), which unfortunately does not include tabulated data. It is important to keep in mind that the dissociation energy of molecular hydrogen is about $4.5 \mathrm{eV}$, therefore 
Table 2 Inelastic processes excited by electron impact with $\mathrm{O}_{2}$ and the associated energy loss

\begin{tabular}{lll}
\hline Excited State & Energy Loss $(\mathrm{eV})$ & Reference \\
\hline Three Body Attachment & & Phelps, Jeon, Magboltz \\
Dissociative Attachment & & Phelps, Jeon, Magboltz \\
Rotation & $0.02,0.02,0.02$ & Phelps, Jeon, Magboltz \\
Vibrational $(v=0 \rightarrow 1)$ & $0.19,0.19,0.193$ & Phelps, Jeon, Magboltz \\
Vibrational $(v=0 \rightarrow 2)$ & $0.38,0.38,0.386$ & Phelps, Jeon, Magboltz \\
Vibrational $(v=0 \rightarrow 3)$ & $0.57,0.57,0.579$ & Phelps, Jeon, Magboltz \\
Vibrational $(v=0 \rightarrow 4)$ & $0.75,0.75,0.772$ & Phelps, Jeon, Magboltz \\
$a^{1} \Delta_{g}$ & $0.977,0.977,0.977$ & Phelps, Jeon, Magboltz \\
$b^{1} \Sigma_{g}^{+}$ & $1.627,1.627,1.627$ & Phelps, Jeon, Magboltz \\
4.5 Loss, $c^{1} \Sigma_{u}^{-}+c^{3} \Delta_{u}$ & $4.5,4.5$ & Phelps, Magboltz \\
6.0 Loss, $A^{3} \Sigma_{u}^{+}$ & $6.0,6.0,6.1$ & Phelps, Jeon, Magboltz \\
8.4 Loss, $B^{3} \Sigma_{u}^{-}$ & $8.4,8.4,8.4$ & Phelps, Jeon, Magboltz \\
9.97 Loss & $9.97,9.97,9.3$ & Phelps, Jeon, Magboltz \\
10.29 Loss & 10.29 & Jeon \\
13.3 Loss & 13.3 & Jeon \\
\hline
\end{tabular}

Table 3 Inelastic processes excited by electron impact with $\mathrm{H}_{2}$ and the associated energy loss

\begin{tabular}{lll}
\hline Excited State & Energy Loss $(\mathrm{eV})$ & Reference \\
\hline Dissociative Attachment & & Magboltz \\
Rotation $(J=0 \rightarrow 2)$ & $0.044,0.0439$ & Phelps, Magboltz \\
Rotation $(J=1 \rightarrow 3)$ & $0.073,0.727$ & Phelps, Magboltz \\
Vibration $(v=0 \rightarrow 1)$ & $0.516,0.516$ & Phelps, Magboltz \\
Vibration $(v=0 \rightarrow 2)$ & $1.0,1.023$ & Phelps, Magboltz \\
Vibration $(v=0 \rightarrow 3)$ & $1.5,1.48$ & Phelps, Magboltz \\
$b^{3} \Sigma_{u}^{+}$, Triplet States & $8.9,8.85$ & Phelps, Magboltz \\
$B^{1} \Sigma_{u}^{+}$ & 11.3 & Phelps \\
$c^{3} \Pi_{u}$ & 11.75 & Phelps \\
$a^{3} \Sigma_{g}^{+}$ & 11.8 & Phelps \\
Singlet States & 12.0 & Magboltz \\
$C^{1} \Pi_{u}$ & 12.4 & Phelps \\
Lyman Alpha & 15.0 & Phelps \\
Rydberg Sum & 15.2 & Phelps \\
Balmer Alpha & 16.6 & Phelps \\
\hline
\end{tabular}

many electronic excitations lead to dissociation. Table 3 lists the various inelastic interactions along with their associated energy loss terms. Figure $2 \mathrm{c}$ shows a plot of the various $\mathrm{H}_{2}$ cross-sections represented as energy loss per distance traveled at sea level as a function of energy. To construct this plot we have taken the cross sections from the Phelps compilation along with an elastic cross section estimated from data compiled by Brunger and Buckman (2002), and a plot in the Tawara et al. (1990a). Figure 2c also shows the relativistic energy loss calculated from the Bethe formula (2.3). 


\subsubsection{Carbon Dioxide}

Both the Phelps and Magboltz compilations cover $\mathrm{N}_{2}$. In 2002, Itikawa published a compilation of his recommended values of the $\mathrm{CO}_{2}$ cross-sections (Itikawa 2002). This work by Itikawa includes tabulations for the total, elastic, momentum transfer, three vibrations $(100,010,001)$, ionization, and the dissociative attachment cross sections. In the conclusion Itikawa acknowledges the omission of electronic states from his compilation saying that they are either small or not known quantitatively. Indeed his review of even the state assignments show little agreement, nor do they agree with the compilations looked at here. $\mathrm{He}$ also discusses the vibrational resonance region around $3.8 \mathrm{eV}$ saying that there are likely significant overtone bands in this region, indicated by the difference between total and elastic cross sections there. This assessment is consistent with the differences seen between the Phelps and Magboltz compilations, which are significant especially in the light of energy loss rather than total cross section. The Magboltz compilation includes 'Polyad' transitions, which we take to be vibrational resonances sometimes referred to as Fermi dyads; these contribute significantly to the energy loss if not so much the total cross section. Table 4 lists the various inelastic interactions along with their associated energy loss terms. Figure $2 \mathrm{~d}$ shows a plot of the various $\mathrm{CO}_{2}$ cross-sections represented as energy loss per distance traveled at sea level as a function of energy. To construct this plot we have taken the cross sections from the Magboltz compilation. Figure $2 \mathrm{~d}$ also shows the relativistic energy loss calculated from the Bethe formula (2.3).

\subsubsection{Methane}

Methane is the only gas we address that is not covered by the Phelps compilation. Magboltz does include it, and there is some additional information in the paper by Kanik et al. (1993). Kanik estimates and tabulates integral elastic, vibrational, and electronic cross sections. Summing over Magboltz cross sections to give an appropriate comparison shows large differences between the compilations, exceeding a factor of 2 in some cases. Tawara et al. (1990b) also published a review in 1990, but does not include tabulated data and does not recommend any electronic transitions. Table 5 lists the various inelastic interactions along with their associated energy loss terms. Figure $2 \mathrm{e}$ shows a plot of the various $\mathrm{CH}_{4}$ crosssections represented as energy loss per distance traveled at sea level as a function of energy. To construct this plot we have taken the cross sections from the Magboltz compilation, as it is the most complete. Figure 2e also shows the relativistic energy loss calculated from the Bethe formula (2.3).

\subsubsection{Helium}

Helium is an important gas because it is commonly used as the standard in relative flow techniques to obtain absolute scattering cross-sections. It has the useful property that only elastic scattering is available below its inelastic threshold of about $19.8 \mathrm{eV}$. Both the Phelps and Magboltz compilations cover Helium. Comments in the Magboltz code claim an accuracy of $0.2 \%$ at all fields. We have not found this level of agreement in the open literature (see e.g. Brunger et al. 1992). Table 6 lists the various inelastic interactions along with their associated energy loss terms. Figure $2 \mathrm{f}$ shows a plot of the various He cross-sections represented as energy loss per distance traveled at sea level as a function of energy. To construct this plot we have taken the cross sections from the Magboltz compilation. Figure $2 \mathrm{f}$ also shows the relativistic energy loss calculated from the Bethe formula (2.3). 
Table 4 Inelastic processes excited by electron impact with $\mathrm{CO}_{2}$ and the associated energy loss

\begin{tabular}{lll}
\hline Excited State & Energy Loss $(\mathrm{eV})$ & Reference \\
\hline Dissociative Attachment & & \\
Asymmetric Stretch, $V(010)$ Bend Mode & $0.083,0.08275$ & Phelps, Magboltz \\
Vibration 2, $V(020)$ Bend Mode Resonance & $0.167,0.15937$ & Phelps, Magboltz \\
$V(100)$ Symmetric Stretch & 0.17211 & Magboltz \\
0.252 Loss, $V(030)+V(110)$ & $0.252,0.251$ & Phelps, Magboltz \\
Vibration 3, $V(001)$ Asymmetric Stretch & $0.291,0.29126$ & Phelps, Magboltz \\
0.339 Loss, Polyad 3 & $0.339,0.335$ & Phelps, Magboltz \\
0.422 Loss, $V(130)+V(210)$ & $0.422,0.422$ & Phelps, Magboltz \\
0.505 Loss, Polyad 4 & $0.505,0.505$ & Phelps, Magboltz \\
Polyad 5 & 0.685 & Magboltz \\
Polyad 6 & 0.825 & Magboltz \\
Polyad 7 & 0.995 & Magboltz \\
Polyad 8 & 1.16 & Magboltz \\
Polyad 9 & 1.32 & Magboltz \\
2.5 Loss, Sum Higher Polyads & $2.5,2.5$ & Phelps, Magboltz \\
3.85 Loss & 3.85 & Phelps \\
7.0 Loss & 7.0 & Phelps \\
Excitation 1 & 7.9 & Magboltz \\
Excitation 2 & 8.9 & Magboltz \\
10.5 Loss, Excitation 3 & $10.5,10.5$ & Phelps, Magboltz \\
Excitation 4 & 12.2 & Magboltz \\
13.3 Loss, Excitation 5 & $13.3,13.2$ & Phelps, Magboltz \\
Excitation 6 & 15.0 & Magboltz \\
\hline
\end{tabular}

Table 5 Inelastic processes excited by electron impact with $\mathrm{CH}_{4}$ and the associated energy loss

\begin{tabular}{lcc}
\hline Excited State & Energy Loss $(\mathrm{eV})$ & Reference \\
\hline Dissociative Attachment & & \\
VIB $V 2+V 4$ & 0.1625 & Magboltz \\
VIB $V 1+V 3$ & 0.3743 & Magboltz \\
VIB HAR1 & 0.544 & Magboltz \\
VIB HAR2 & 0.736 & Magboltz \\
EXC DISOCIATION1 & 9.000 & Magboltz \\
EXC DISOCIATION2 & 10.000 & Magboltz \\
EXC DISOCIATION3 & 11.000 & Magboltz \\
EXC DISOCIATION4 & 11.800 & Magboltz \\
\hline
\end{tabular}

\subsection{Input to Relativistic Boltzmann Equation}

In the Fokker-Planck treatment described in Sect. 2.1 the electron encounters with gas molecules are represented as a mean drag (energy loss) plus a scattering in angle. The gas parameters that enter into this formulation are the mean charge $Z$ and the mean excitation energy $I$. Values appropriate for planetary gases are listed in Table 7. The resulting energy loss is plot- 
Table 6 Inelastic processes excited by electron impact with He along and associated energy loss

Table 7 Mean charge and excitation energy for planetary gases. Data taken from ICRU (1993)

\begin{tabular}{lll}
\hline Excited State & Energy Loss $(\mathrm{eV})$ & Reference \\
\hline Triplet State, 19.8 Loss & $19.82,19.8$ & $\begin{array}{l}\text { Phelps, Magboltz } \\
\text { Magboltz }\end{array}$ \\
\hline
\end{tabular}

\begin{tabular}{lll}
\hline GAS & Mean Charge $(Z)$ & Mean Excitation Energy $(I$ in eV $)$ \\
\hline $\mathrm{H}_{2}$ & 2 & 19.2 \\
$\mathrm{~N}_{2}$ & 14 & 82.0 \\
$\mathrm{O}_{2}$ & 16 & 95.0 \\
$\mathrm{CH}_{4}$ & 10 & 41.7 \\
$\mathrm{CO}_{2}$ & 22 & 85.0 \\
$\mathrm{He}$ & 2 & 41.8 \\
\hline
\end{tabular}

ted in Figs. 2a-f. The corresponding numbers for gas mixtures are found by weighting the values for the individual constituents by the gas fractional concentration. We note that the energy loss calculated from the Bethe formula and that calculated from the tabulated cross sections do not always agree. The source of this disagreement, and how best to address it, is not yet clear to us.

\section{Solutions of the Kinetic Equations}

\subsection{Numerical Solution Techniques}

\subsubsection{Relativistic Boltzmann Equation}

In this section we describe the numerical solution of the relativistic Boltzmann kinetic equation for the electron distribution function, $f(t, p, \mu)$, in a uniform atmosphere and with an applied, fixed, external electric field. Recall that $p$ is the magnitude of the electron momentum, $\mu$ is the cosine of the angle between the electric field and the momentum vector, and $\delta_{0}$ is the ratio of the electric field strength to the runaway threshold field or the overvoltage. This parameter represents the natural scaling for runaway breakdown and has been used extensively in the literature. It is related to the scaled electric field $E / N_{m}$ expressed in Townsend or Td (where $N_{m}$ is the molecular gas density and $1 \mathrm{Td}=10^{-21} \mathrm{~V} \mathrm{~m}^{2}$ ) in air by $E / N_{m}=8 \cdot \delta_{0}$. The corresponding formula for the other planets can be derived from the values given in the last column of Table 8 . From $f(t, p, \mu)$, we can compute the avalanche time, the average electron beam energy, and the spread in the beam energy. We recast the relativistic Boltzmann equation into a form, through a change of variables, that allows one to use the machinery of computational fluid dynamics for its numerical solution. The new, normalized, variables are:

$$
\begin{aligned}
& p=p / m c ; \quad F=F_{D} / F_{D, \min } ; \quad \varepsilon=\varepsilon / m c^{2} ; \\
& \rho=p f ; \quad t=\left(F_{D, \min } / m c\right)\left(N_{m} / N_{m}^{0}\right) t .
\end{aligned}
$$

With these variables, (2.1) and (2.2) can be written as:

$$
\frac{\partial \rho}{\partial t}+\nabla \cdot(\rho v)=\frac{\partial}{\partial \mu}\left\{\Gamma \frac{\partial \rho}{\partial \mu}\right\}+p S_{i o n}
$$


Table 8 Relative concentrations of the constituent gases that make up the atmosphere's of the major planets as well as Titan and Triton are listed along with the corresponding mean molecular charge $(Z)$, mean excitation energy $(I)$, and threshold electric field at STP. The scaled electric field $\left(E / N_{m}\right)$ in Td is also provided in each case. The asterisks indicate the specific gas mixtures (planets or moons) for which detailed kinetic calculations have been performed

\begin{tabular}{|c|c|c|c|c|}
\hline $\begin{array}{l}\text { Planet } \\
* \text { Modeled }\end{array}$ & Gas Mixture & $\begin{array}{l}\text { Mean Charge } \\
(Z)\end{array}$ & $\begin{array}{l}\text { Mean Excitation } \\
\text { Energy } I(\mathrm{eV})\end{array}$ & $\begin{array}{l}\text { Runaway Threshold } \\
E_{t h}(\mathrm{kV} / \mathrm{m})\end{array}$ \\
\hline Venus* & $\begin{array}{l}96.5 \% \mathrm{CO}_{2} \\
3.5 \% \mathrm{~N}_{2}\end{array}$ & 21.7 & 85 & $320(11.9 \mathrm{Td})$ \\
\hline Earth* & $\begin{array}{l}78 \% \mathrm{~N}_{2} \\
21 \% \mathrm{O}_{2} \\
1 \% \mathrm{Ar}\end{array}$ & 14.5 & 80.5 & $215(8.00 \mathrm{Td})$ \\
\hline Mars & $\begin{array}{l}95.5 \% \mathrm{CO}_{2} \\
2.8 \% \mathrm{~N}_{2} \\
1.7 \% \mathrm{Ar}\end{array}$ & 21.7 & 85 & $320(11.9 \mathrm{Td})$ \\
\hline Jupiter* & $\begin{array}{l}89 \% \mathrm{H}_{2} \\
10.9 \% \mathrm{He} \\
0.1 \% \mathrm{CH}_{4}\end{array}$ & 2.01 & 19.2 & $34.1(1.27 \mathrm{Td})$ \\
\hline Saturn & $\begin{array}{l}96.3 \% \mathrm{H}_{2} \\
3.6 \% \mathrm{He} \\
0.1 \% \mathrm{CH}_{4}\end{array}$ & 2.01 & 19.2 & $34.1(1.27 \mathrm{Td})$ \\
\hline Neptune & $\begin{array}{l}80 \% \mathrm{H}_{2} \\
18.5 \% \mathrm{He} \\
1.5 \% \mathrm{CH}_{4}\end{array}$ & 2.12 & 19.2 & $36.0(1.34 \mathrm{Td})$ \\
\hline Uranus & $\begin{array}{l}82.5 \% \mathrm{H}_{2} \\
15.2 \% \mathrm{He} \\
5 \% \mathrm{CH}_{4}\end{array}$ & 2.18 & 19.2 & $37.0(1.38 \mathrm{Td})$ \\
\hline Triton* & $\begin{array}{l}95 \% \mathrm{~N}_{2} \\
5 \% \mathrm{CH}_{4}\end{array}$ & 13.8 & 82 & $204(7.59 \mathrm{Td})$ \\
\hline Titan & $\begin{array}{l}95 \% \mathrm{~N}_{2} \\
5 \% \mathrm{CH}_{4}\end{array}$ & 13.8 & 82 & $204(7.59 \mathrm{Td})$ \\
\hline
\end{tabular}

where the effective velocities in $(p, \mu)$ space are

$$
v_{\mu}=-\frac{\delta_{0}\left(1-\mu^{2}\right)}{p}, \quad v_{p}=-\left(F+\mu \delta_{0}\right)
$$

and the diffusion coefficient, due to elastic scattering, is

$$
\Gamma=\frac{(1+Z / 2)}{4} \frac{F}{\gamma} \frac{\left(1-\mu^{2}\right)}{p} .
$$

We have now reduced the problem to solving a continuity equation with a diffusion term and source term. The effective velocity field is constant for a given electric field strength. We use a finite volume, cell centered, time explicit, spatially second order accurate algorithm from the CAVEAT code (Addessio et al. 1992) to solve this equation. The finite volume solution requires a discretization of the ionization term over a finite volume element. Our code runs 
were initialized with an isotropic distribution of electrons having a specified mean energy, typically around several $\mathrm{MeV}$ though other values were tested to ensure that the distribution function evolved to the same steady state form. The model was run to a final time such that the distribution function was no longer changing, or was self-similar in the cases where the distribution function magnitude or electron density continues to change due to the runaway avalanche. Details of the solution can be found in Symbalisty et al. (1998).

\subsubsection{Non-relativistic Boltzmann Equation}

The numerical method used to solve the non-relativistic Boltzmann equation revolves around the technique described above and in Symbalisty et al. (1998). We first choose a new independent variable $\rho=v f$ and then recast the advective terms in (2.7) into a form that is equivalent to the usual hydrodynamic equations (see Symbalisty et al. 1998 for details). Then we use the well-known and well-tested numerical solution algorithms from computational fluid dynamics to derive an operator that acts on $\rho$ and represents the change in $\rho$ due to the left hand side of (2.7). Cross sections are weighted according to the number concentration presented in Table 8. The collision term (2.8) is discretized and an operator that acts on $\rho$ at each cell in our grid is derived. The final solution is obtained by expressing the modified distribution function $\rho$ as a vector with each element representing a cell defined by a specific momentum and angle in our grid. The flow and encounter terms were then formulated as matrices within the same dimensional space. The encounter matrix/operator was notated $A$, and the flow matrix/operator as $S$. The differential equation was then solved using an explicit time stepping method, with a time-step picked to ensure that the change of velocity remains smaller than our smallest grid volume in velocity space, i.e., satisfies the Courant condition. Thus, we rearrange the Boltzmann equation to:

$$
\rho(t+\Delta t)=\rho(t) \times(1+A \Delta t-E S \Delta t) .
$$

$E$ is again the applied field.

The model initializes with an isotropic distribution of electrons having a specified mean energy, typically this value was $0.38 \mathrm{eV}$ though other values were tested to ensure that the distribution function evolved to the same steady state form regardless of the starting energy. The model was run to a final time such that the distribution function was no longer changing, or was self-similar in the cases where the distribution function magnitude continues to change due to ionization or attachment. The model was run with all terms normalized to Standard Temperature and Pressure (STP) equivalents, but the output could be adapted to any altitude by rescaling the length $(L)$ and time scales $(\tau)$ such that $N L=$ constant and $N \tau=$ constant and applying an additional correction for the three body attachment rate (in the case of oxygen and air). Thus, velocity and energy have no scaling factor while rates and accelerations (e.g., $E$ ) are proportional to the density $(N)$ and hence the utility of the scaled parameter $E / N$ (i.e., independent of gas density). Though we have all the mechanisms in place to employ differential cross sections, and we intend to do so in the future, we are currently assuming all processes to be isotropic.

\subsection{Results}

Solutions of both the relativistic (runaway breakdown regime) and non-relativistic (conventional breakdown or swarm regime) Boltzmann equations were obtained for gas mixtures relevant to a number of planetary and lunar atmospheres. The relative concentrations of the 
Fig. 3 Electron energy loss rate or dynamical friction force as a function of electron energy for Venus, Earth, Triton, and Jupiter

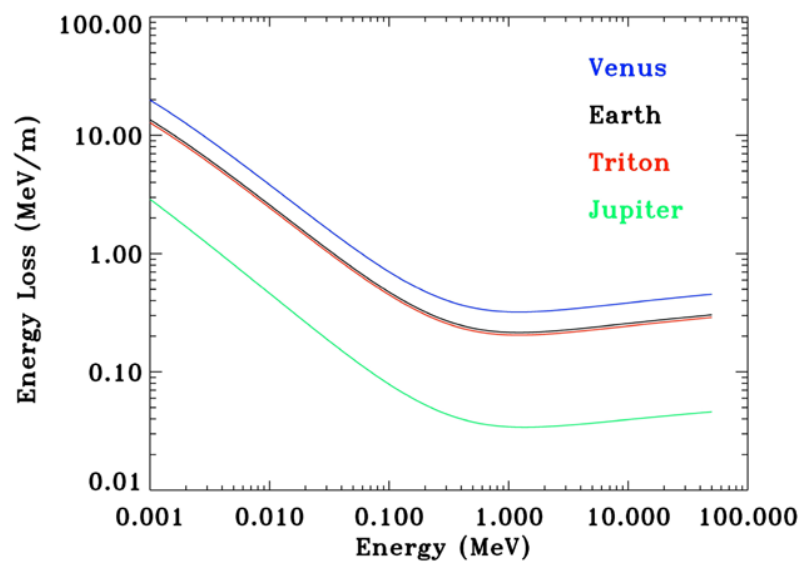

constituent gases that make up the atmosphere's of the major planets as well as Titan and Triton are listed in Table 8 along with the corresponding mean molecular charge $(Z)$, mean excitation energy $(I)$, and threshold electric field at STP. Detailed calculations were performed for Venus (similar to Mars), Earth, Jupiter (representing the gas giants), and Triton (identical to Titan) as indicated by the asterisks in Table 8 . The results are presented below for STP and for a range of scaled values of the electric field $\left(E / N_{m}\right)$ given in Td. The electron distribution function was evolved in time until a self-similar solution was obtained.

\subsubsection{Runaway Breakdown Regime}

One of the primary inputs to the relativistic Boltzmann equation is the dynamical friction force or drag, $F_{D}$, resulting from electron-gas interactions. A plot showing the energy dependence of $F_{D}$ for Venus, Earth, Triton, and Jupiter is provided in Fig. 3. Immediately evident from this plot as from Table 8 is the fact that the local minimum around $1.4 \mathrm{MeV}$ (or the runaway threshold) is much lower in magnitude for the gas giants (Jupiter) than for Earth (by a factor of $\sim 6.3$ ) or the other planets and moons. Thus, runaway breakdown is more likely to be initiated on Jupiter provided the same electric field environments can be attained.

Table 9 lists the avalanche time $\tau$, the average beam energy $\langle\varepsilon\rangle$, and the spread (or standard deviation) $\sigma$ in the beam energy derived from solutions of the relativistic Boltzmann equations for a number of electric field strengths and for the Earth at 1 atmosphere of pressure. The final time of the simulation, TMAX, is also listed. TMAX is always greater than 5 times the avalanche time, in order to allow a similarity solution to develop for the electron distribution function. The avalanche time is found to equilibrate 2 to 3 times faster than the average beam energy.

Similar calculations were performed for Venus, Jupiter, and Triton. The results are shown in Figs. 4a-c where the avalanche time, mean energy, and spread in energy, are plotted as a function of the scaled electric field $(E / N)$ in Td, respectively. One key result is that the avalanche time is smaller for Jupiter than for Earth by large factors at low $E / N$ and by factors of 2-3 at high $E / N$, in agreement with the findings of Dwyer et al. (2006). The indication again is that runaway breakdown initiates more easily and proceeds faster on the gas giants for the same applied electric field. In addition, the mean energy and spread in energy tends to be smaller. As a result we can expect diagnostics such as gamma ray flashes from bremsstrahlung radiation produced by a runaway discharge on Jupiter to be 
Table 9 The avalanche time $\tau$, average beam energy $\langle\varepsilon\rangle$, and the spread (or standard deviation) $\sigma$ in the beam energy are listed for a number of scaled electric field strengths $E / N$ or overvoltages $\delta_{0}$. These results were obtained from solutions of the relativistic Boltzmann equation for the Earth at STP.

The final time of the simulation, TMAX, is also listed

\begin{tabular}{llrcll}
\hline$E / N(\mathrm{Td})$ & $\delta_{0}$ & TMAX $(\mathrm{ns})$ & $\tau(\mathrm{ns})$ & $\langle\varepsilon\rangle(\mathrm{MeV})$ & $\sigma(\mathrm{MeV})$ \\
\hline 15 & 1.85 & 1000 & 194 & 6.91 & 10.8 \\
16.22 & 2.0 & 900 & 158 & 7.05 & 11.3 \\
20 & 2.466 & 600 & 100 & 7.24 & 12.1 \\
24.33 & 3.0 & 435 & 70.8 & 7.34 & 12.5 \\
28.39 & 3.5 & 300 & 55.4 & 7.35 & 12.5 \\
32.44 & 4.0 & 279 & 45.5 & 7.39 & 12.8 \\
35 & 4.316 & 248 & 40.8 & 7.40 & 12.9 \\
40.55 & 5.0 & 200 & 33.3 & 7.39 & 12.9 \\
50 & 6.165 & 140 & 25.3 & 7.34 & 12.8 \\
56.77 & 7.0 & 125 & 21.5 & 7.32 & 12.9 \\
64.9 & 8.0 & 110 & 18.2 & 7.26 & 12.9 \\
72.99 & 9.0 & 95 & 15.7 & 7.20 & 12.8 \\
75 & 9.248 & 80 & 15.2 & 7.16 & 12.6 \\
81.10 & 10.0 & 70 & 13.8 & 7.10 & 12.4 \\
100 & 12.33 & 60 & 10.7 & 6.92 & 12.3 \\
125 & 15.41 & 55 & 7.93 & 6.64 & 11.9 \\
150 & 18.50 & 50 & 6.24 & 6.36 & 11.5 \\
200 & 24.66 & 25 & 4.23 & 5.82 & 10.6 \\
\hline
\end{tabular}

significantly different. In particular, the spectrum of the gamma rays would be softer on Jupiter than on Earth.

Results for the electron distribution at high energies for the various planets and moons and for $E / N=20 \mathrm{Td}$ and $E / N=200 \mathrm{Td}$ are presented in Figs. 5 and 6 , respectively. Clearly evident in these plots is the collimation of the electrons anti-parallel to the electric field. The collimation increases significantly at the larger field strength. Also evident is that the electrons are significantly more aligned with the electric field in the Jovian atmosphere for the same field strength. This result indicates that the gamma emissions produced by a runaway discharge on Jupiter would be significantly more forward directed (along the electric field) for the same electric field.

\subsubsection{Non-relativistic Regime}

Here we present representative results from our kinetic calculations of electron swarms in various pure gases and compare them to various experimental determinations.

\section{Summary of Planets}

Figures 7, 8, 9, 10 show that we are accurately representing the bulk properties of the electron swarm within this range of applied fields. We do not have the data necessary to benchmark calculations of the gas mixtures that mirror the planetary atmospheres. Nevertheless we have performed those calculations and some of the results are presented below. Figures 11 and 12 show the self-similar distribution functions obtained for calculations of the atmospheres of Jupiter, Triton, and Venus at applied fields of 20 and 200 Townsend respectively. At 20 Td, Fig. 11, the applied electric field lies below the ionization threshold for all gas mixtures and we see that the mean electron energy and shape of the distribution function 
Fig. 4 a Avalanche time in ns, b Mean electron energy, and c Electron energy spread in $\mathrm{MeV}$ as a function of scaled electric field $(E / N)$ in Td for Venus, Earth, Triton, and Jupiter

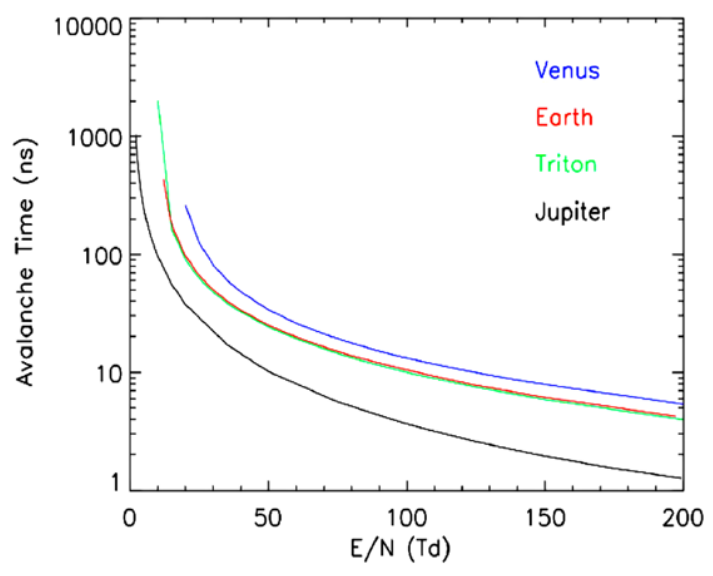

a

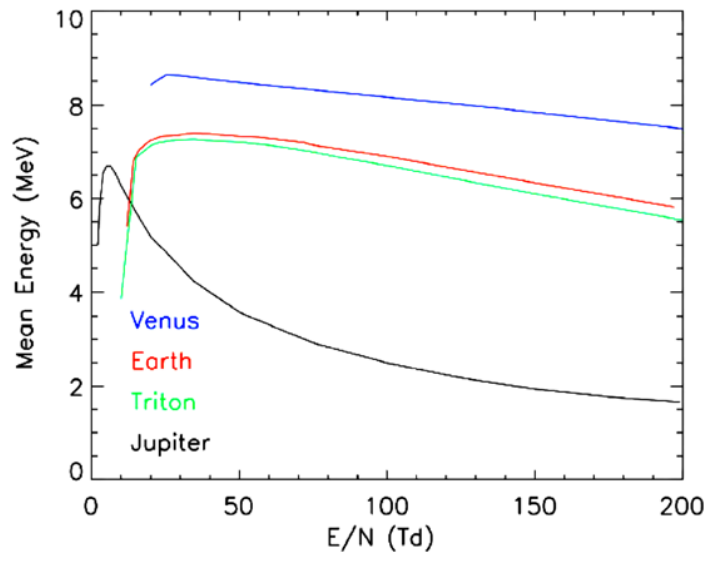

b

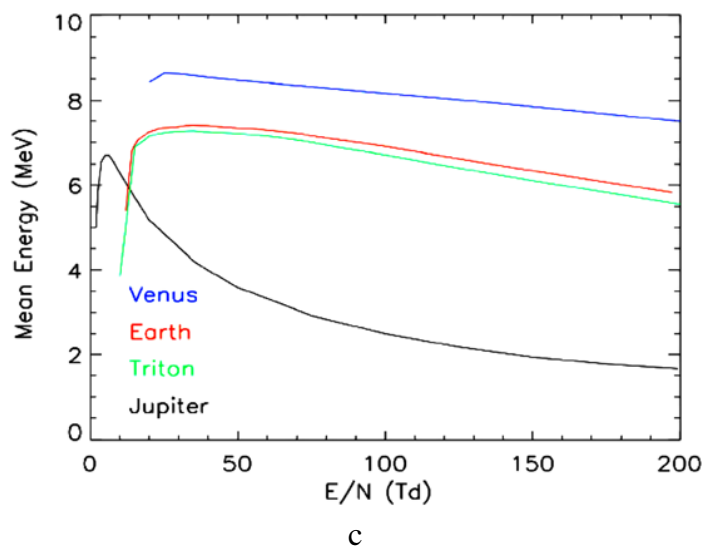



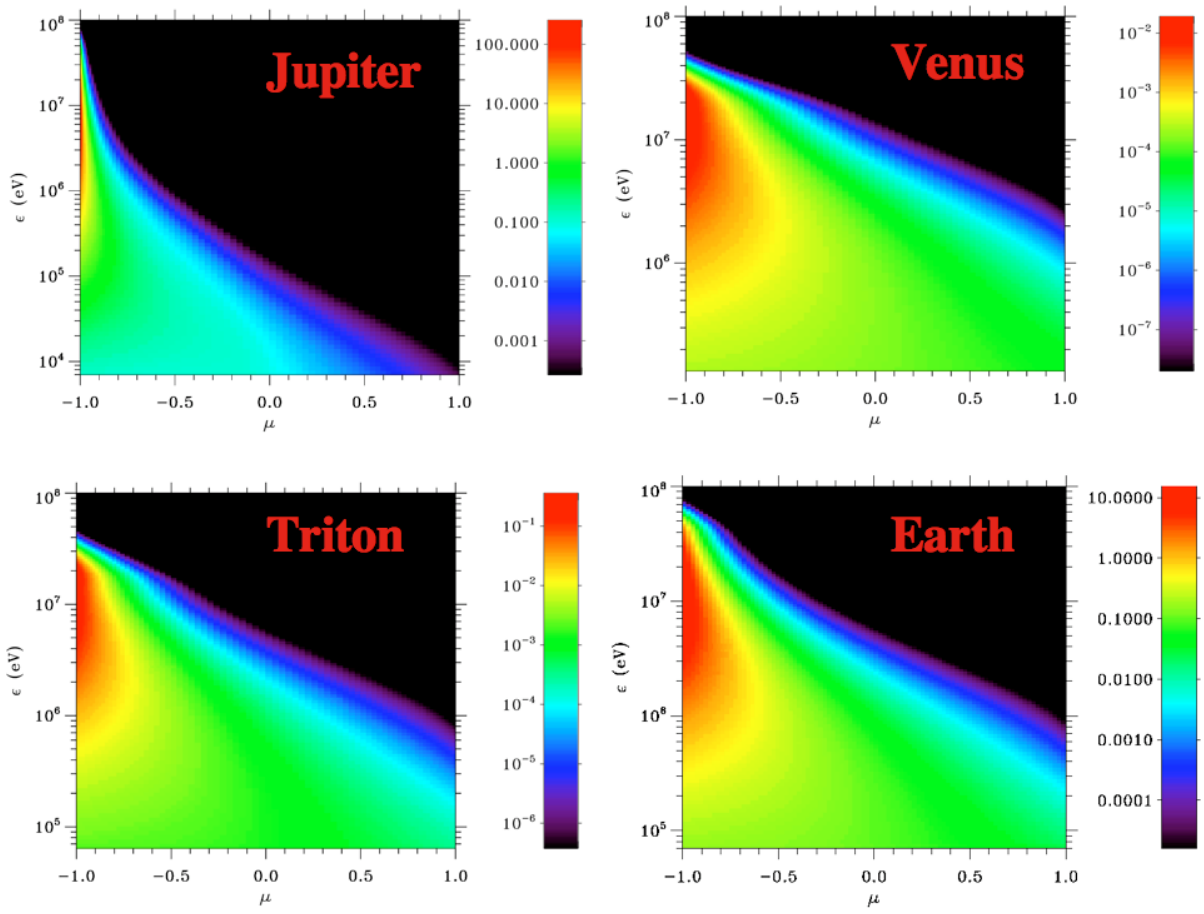

Fig. 5 Two-dimensional plots of the electron distribution functions as a function of the cosine of the angle $\mu$ between the electric field and the electron momentum and as a function of the electron energy $\varepsilon$ for Venus, Earth, Triton, and Jupiter and for $(E / N)=20 \mathrm{Td}$

depends strongly on the details of the vibrational collision cross-sections around several $\mathrm{eV}$ (plotted along side the distribution functions for reference). In particular, the distribution function is depleted by the energy loss associated with vibrational excitation of $\mathrm{N}_{2}$ (Triton) and $\mathrm{CO}_{2}$ (Venus) but not $\mathrm{H}_{2}$ (Jupiter). See also Figs. 2a-f. At $200 \mathrm{Td}$, Fig. 12, the electric field exceeds the threshold for ionization in all cases and we see a splitting of the distribution function in the case of $\mathrm{N}_{2}$ and $\mathrm{CO}_{2}$ caused by the strong energy loss associated with vibrational transitions. In the case of Jupiter we see a broader, more energetic distribution function peaked in the direction of the electric field. Note that the energy scales vary from plot to plot.

\section{Earth's High-Altitude Discharges}

In this section we briefly describe the recently discovered discharge phenomena of transient luminous events (TLEs) and terrestrial gamma-ray flashes (TGFs). Sprites are covered in more detail because of the large body of relevant data that exists and because of their close tie to well known discharge processes. Examples of diagnostics that can and have provided information about the physical nature and origin of sprites is included. The phenomena of TLEs and TGFs may well have counterparts on other planetary systems and it is important to understand their characteristics as manifested on Earth, their impact on the terrestrial environment, and how to detect them. 

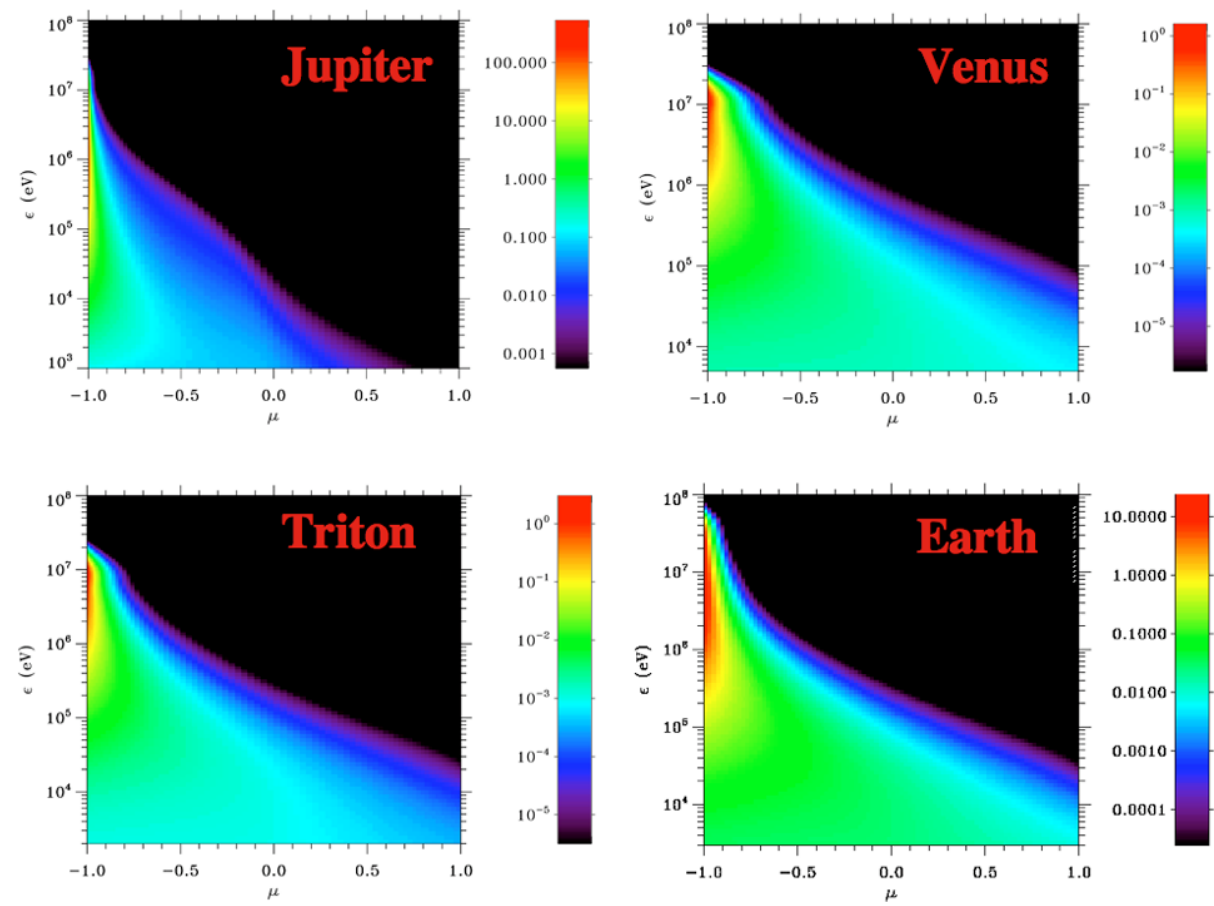

Fig. 6 Two-dimensional plots of the electron distribution functions as a function of the cosine of the angle $\mu$ between the electric field and the electron momentum and as a function of the electron energy $\varepsilon$ for Venus, Earth, Triton, and Jupiter and for $(E / N)=200 \mathrm{Td}$
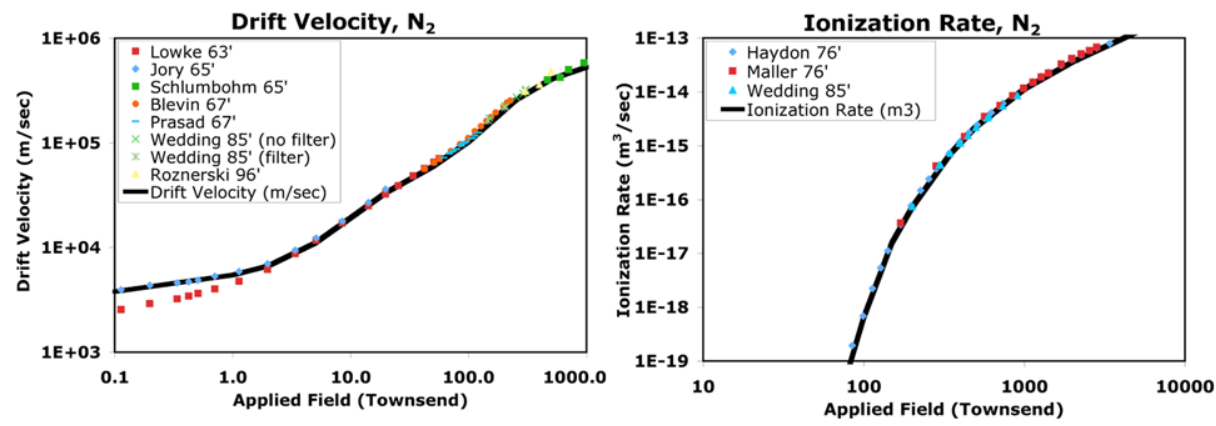

Fig. 7 Calculated Drift Velocities and Ionization rates in $\mathrm{N}_{2}(\mathrm{~g})$ (at STP) plotted along with experimental determinations

TLEs are believed to result from a discharge process that develops in the quasielectrostatic fields that appear in the upper atmosphere following a cloud-to-ground lightning discharge in which large quantities of positive charge $(\sim 100$ to several hundred Coulombs) and occasionally negative charge are transferred to ground. The optical measurements of TLEs (over 10000 events have been recorded) both from the ground (Lyons 2006) and from orbit (e.g., ISUAL on FORMOSAT-2, Mende et al. 2006) have resulted in the phenomenological identification of a large number of processes that have been organized 

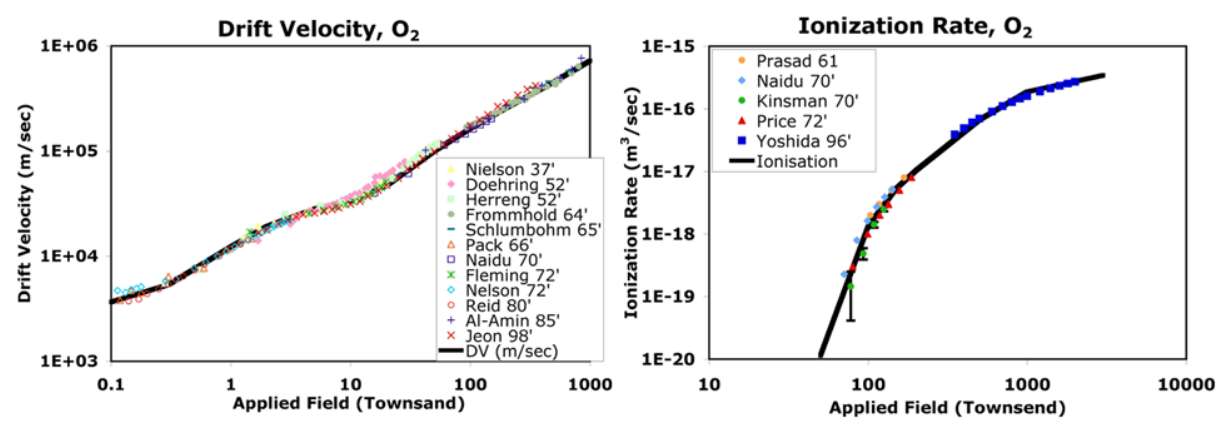

Fig. 8 Calculated Drift Velocities and Ionization Rates in $\mathrm{O}_{2}(\mathrm{~g})$ (at STP) plotted along with experimental determinations
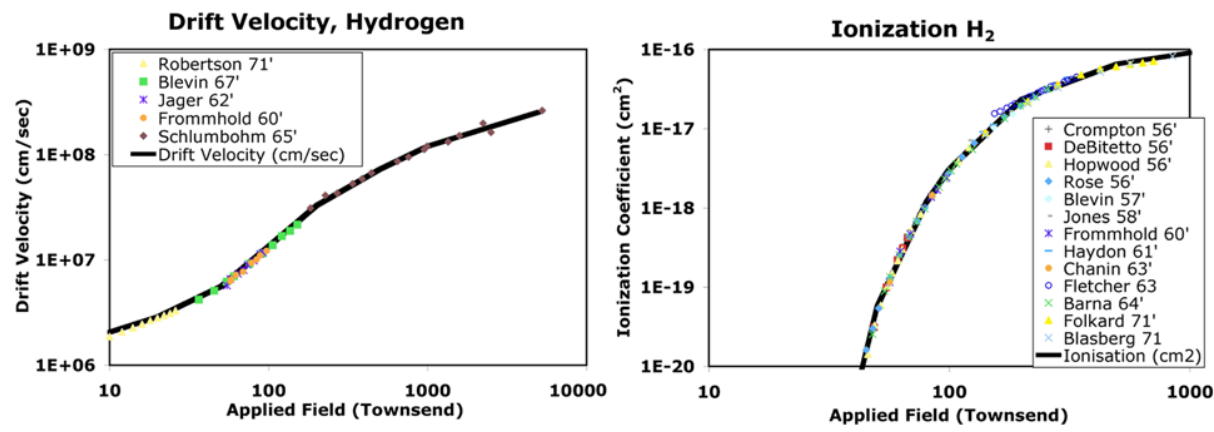

Fig. 9 Calculated Drift Velocities and Ionization Rates in $\mathrm{H}_{2}$ (g) (at STP) plotted along with experimental determinations
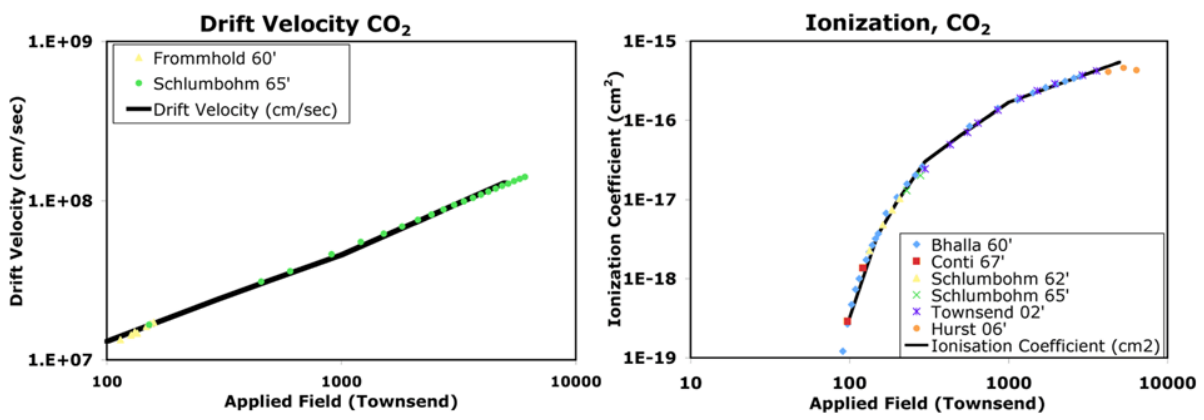

Fig. 10 Calculated Drift Velocities and Ionizations Rates in $\mathrm{CO}_{2}(\mathrm{~g})$ (at STP) plotted along with experimental determinations

into a taxonomy that includes many descriptive and non-descriptive names such as column and carrot sprites, angels, trolls, jets, giant jets, halos, elves, and beads (i.e. Sentman and Wescott 1993; Lyons et al. 2003a, 2003b; Lyons 2006; Cummer et al. 2006a, 2006b; Asano et al. 2008). The relevant scale lengths range from tens of meters to tens and hundreds of kilometers while the temporal scales range from hundreds of $\mu$ s to hundreds of ms. The total energy dissipated in individual events is generally around tens of MJ with power mea- 


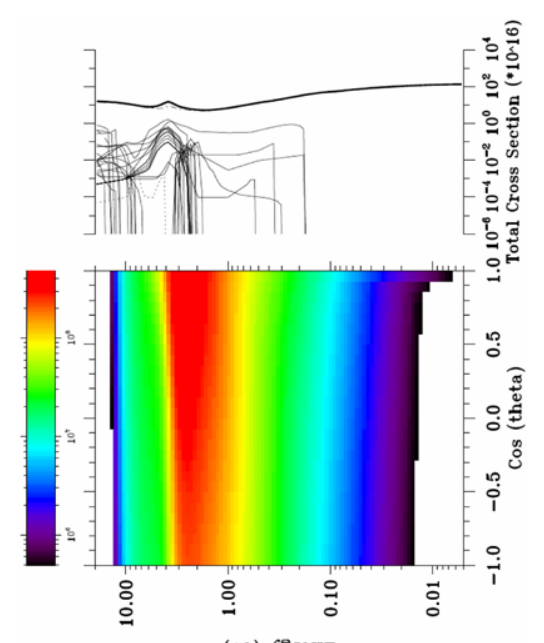

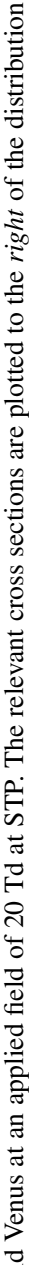

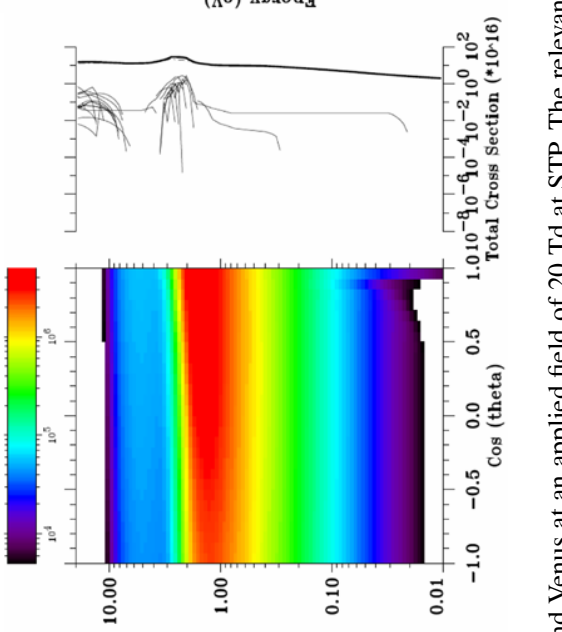

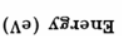

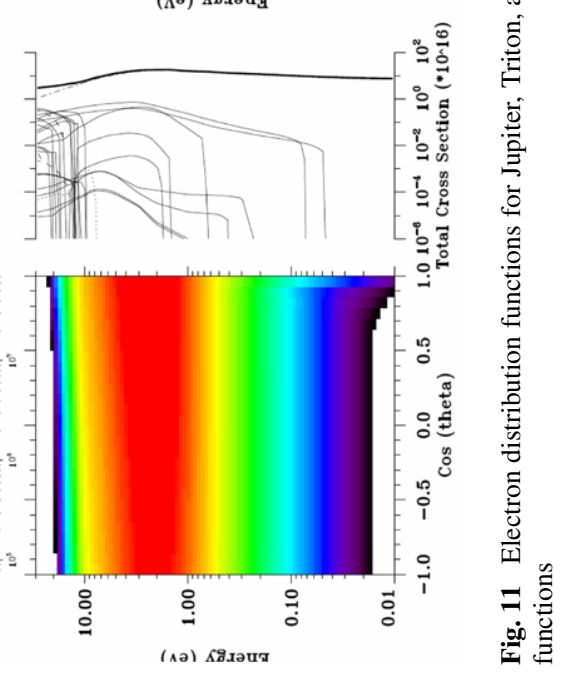




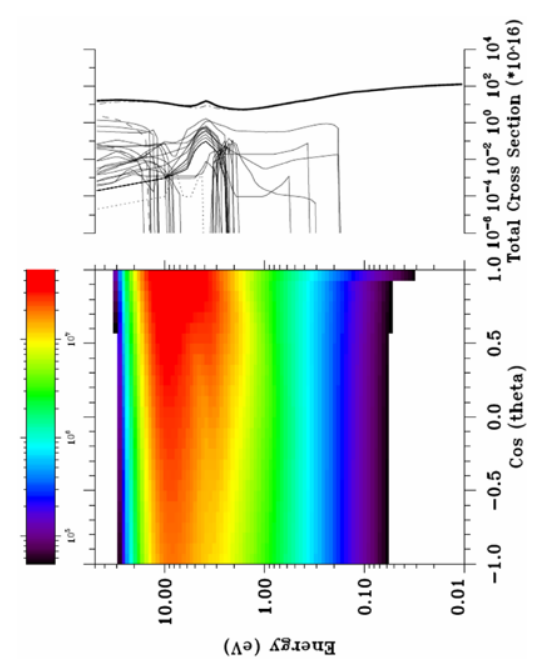

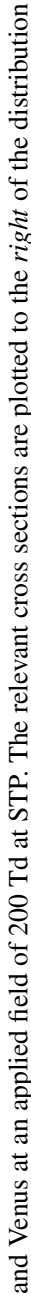
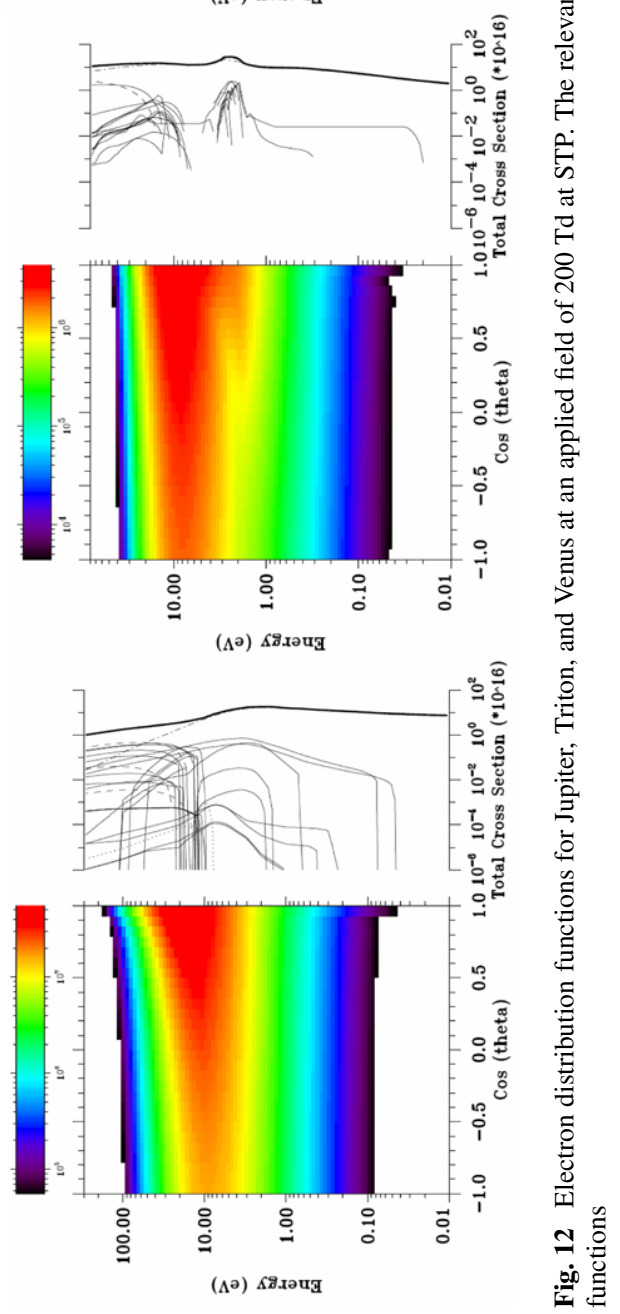
sured in gigawatts (Heavner et al. 2000). The electron densities generated in the mesosphere can exceed $10^{6} \mathrm{~cm}^{-3}$ (cf., Liu and Pasko 2004) while the individual electron energies in these postulated electrical discharges can range from a few $\mathrm{eV}$ to tens of $\mathrm{MeV}$ and produce emissions throughout the electromagnetic spectrum. While our understanding of certain aspects of TLEs, e.g., elves, halos, and sprites, is progressing or in hand, many of the details, especially concerning their chemical effects in the atmosphere (e.g., Enell et al. 2008; Sentman et al. 2008), are not. Dedicated satellite missions are presently in planning to further study energetics and chemical effects of sprites and other transient luminous events on the upper atmosphere (e.g., Blanc et al. 2007).

TGFs are thought to be a manifestation of a RB process that occurs inside thunderstorms. These energetic phenomena were first discovered by the Burst and Transient Source Experiment (BATSE) on the Compton Gamma Ray Observatory (CGRO, Fishman et al. 1994) and are presently being monitored by the Reuven Ramaty High Energy Solar Spectroscopic Imager (RHESSI) satellite, which to date has observed some 10-20 TGFs per month (Smith et al. 2005). The time duration of individual events ranges from hundreds of $\mu \mathrm{s}$ to ms. The geographical distribution of TGFs roughly corresponds to the geographical distribution of lightning over continents at low latitude and also to the distribution of sprites (Christian et al. 2003; Chen et al. 2005). However TGF emissions are rarely detected over the Southern US where many sprites are observed at ground level (Smith et al. 2005). TGF spectra measured by RHESSI reveal energies up to $30 \mathrm{MeV}$ (Smith et al. 2005), in agreement with energies predicted by the RB mechanism triggered by cosmic rays (Roussel-Dupré and Gurevich 1996; Roussel-Dupré et al. 2005; Dwyer and Smith 2005; Østgaard et al. 2008). The low energy part of the TGF spectrum (below $100 \mathrm{keV}$ ) is most sensitive to the TGF emission altitude, due to the large attenuation of low energy gamma rays in the atmosphere. However, in the case of RHESSI, which was not designed to look for TGFs, the instrument response to terrestrial events precludes using the low energies to ascertain source altitude. The analysis of the RHESSI spectra around $1 \mathrm{MeV}$ suggests that their source is in the range of 15-21 km, implying that thunderstorms and not sprites may initiate TGFs (Dwyer and Smith 2005). A recent analysis, however, of BATSE spectra shows that the source of BATSE TGFs could extend continuously from $15 \mathrm{~km}$ to $60 \mathrm{~km}$ altitude rather than in a narrow altitude range (Østgaard et al. 2008). The possibility of two kinds of TGFs corresponding to low and high altitude sources can be envisaged. A lightning leader as a source of TGFs is predicted by Moss et al. (2006) who show that thermal electrons can be accelerated in the leader streamer zone up to energies of several hundreds of $\mathrm{keV}$ and possibly up to several tens of MeV. This mechanism then predicts that some TGFs can be produced by high altitude leader processes.

\subsection{General Phenomenology of Sprites}

Sprites are large luminous discharges, which appear in the altitude range $<40-90 \mathrm{~km}$ above large thunderstorms typically following intense positive cloud-to-ground lightning discharges (Sentman et al. 1995; Boccippio et al. 1995; see also Fig. 13). The evolution of these discharges and their optical emissions are strongly dependent on the details of the electron distribution functions associated with the coupled, self-consistent electric fields that drive the process. The kinetic calculations and methodology outlined above are essential to modeling and understanding these phenomena. Below we describe the observations.

Recent telescopic imaging of sprites at standard video rates (i.e. with $16 \mathrm{~ms}$ time resolution) revealed an amazing variety of generally vertical fine structure with transverse spatial scales ranging from tens to a few hundreds of meters (Gerken et al. 2000; 


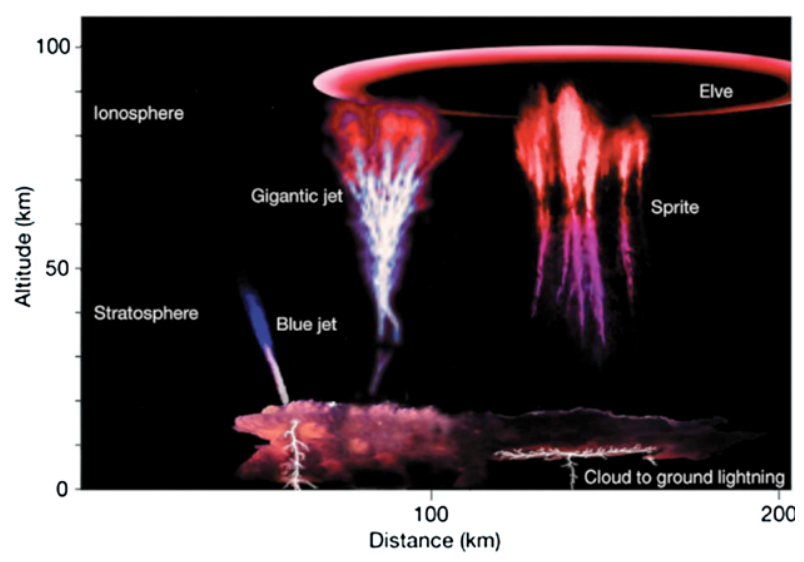

Fig. 13 Lightning related TLEs. Several types of TLEs are known, and some examples are shown here: relatively slow-moving fountains of blue light, known as 'blue jets', which emanate from the top of thunderclouds up to an altitude of $40 \mathrm{~km}$ (Wescott et al. 1995; Lyons et al. 2003a), 'sprites' that develop at the base of the ionosphere and move rapidly downwards at speeds up to $10000 \mathrm{~km} \mathrm{~s}^{-1}$ (Sentman et al. 1995; Lyons 1996; Stanley et al. 1999), 'elves', which are lightning induced flashes that can spread over $300 \mathrm{~km}$ laterally (Fukunishi et al. 1996; Inan et al. 1997; Kuo et al. 2007), and upward moving 'gigantic jets', which establish a direct path of electrical contact between thundercloud tops and the lower ionosphere (Pasko et al. 2002; Su et al. 2003; Pasko 2003). Reprinted from (Pasko 2003) with permission from Nature

Gerken and Inan 2002, 2003, 2005). First high-speed (1 ms) telescopic imaging of sprites has been reported indicating that streamer-like formations in sprites rarely persist for more than 1-2 ms (Marshall and Inan 2005, 2006). Also recently, it has been demonstrated that sprites often exhibit a sharp altitude transition between the upper diffuse and the lower highly structured regions (Gerken and Inan 2002, 2003; Stenbaek-Nielsen et al. 2000; Pasko and Stenbaek-Nielsen 2002). Many sprites are observed with an amorphous diffuse glow at their tops, the so-called sprite 'halo' (cf., Gerken and Inan 2003; Barrington-Leigh et al. 2001).

The appearance of fine structure in sprites has been interpreted in terms of positive and negative streamer coronas, which are considered as scaled analogs of small-scale streamers, which exist at high atmospheric pressures at ground level (cf., Pasko et al. 1998; Sentman et al. 2008). Streamers are filamentary plasma structures, which can initiate spark discharges in relatively short (several $\mathrm{cm}$ ) gaps at near ground pressures in air and which are commonly utilized in applications such as ozone production and pollution control (Raizer 1991; van Veldhuizen 2000) and references cited therein), and also represent important components involved in the triggering of combustion in spark ignition engines (Tardiveau et al. 2001; Tardiveau and Marode 2003). An excellent recent review of various applications of streamers is provided in Ebert et al. (2006). In ground air pressure applications a typical transverse scale of individual streamer filaments is a fraction of a millimetre (Pancheshnyi et al. 2005; Briels et al. 2005). It is quite remarkable that the filamentary structures observed in sprites (Gerken and Inan 2005) are the same phenomenon known as streamer discharges at atmospheric pressure, only scaled by reduced air density at higher altitudes (Pasko et al. 1998; Liu and Pasko 2004, 2006; Pasko 2006). These aspects of sprite phenomenology are important for interpretation of optical emissions observed from them.

Table 10 (Liu et al. 2006) summarizes emissions documented in sprites. These include the First Positive $\left(1 \mathrm{PN}_{2}\right)$ and Second Positive $\left(2 \mathrm{PN}_{2}\right)$ band systems of $\mathrm{N}_{2}, \mathrm{~N}_{2}$ LymanBirge-Hopfield ( $\mathrm{LBH})$ band system and the First Negative band system of $\mathrm{N}_{2}^{+}\left(1 \mathrm{NN}_{2}^{+}\right)$. 
Table 10 Summary of observed emissions from sprites (Liu et al. 2006)

\begin{tabular}{lllll}
\hline $\begin{array}{l}\text { Emission band } \\
\text { System }\end{array}$ & Transition & $\begin{array}{l}\text { Excitation energy } \\
\text { threshold }(\mathrm{eV})\end{array}$ & $\begin{array}{l}\text { Lifetime at } \\
70 \mathrm{~km} \text { Alt. }\end{array}$ & $\begin{array}{l}\text { Quenching } \\
\text { Alt. }(\mathrm{km})\end{array}$ \\
\hline $1 \mathrm{PN}_{2}$ & $\mathrm{~N}_{2}\left(B^{3} \Pi_{g}\right) \rightarrow \mathrm{N}_{2}\left(A^{3} \Sigma_{u}^{+}\right)$ & $\sim 7.35$ & $5.4 \mu \mathrm{s}$ & $\sim 53$ \\
$2 \mathrm{PN}_{2}$ & $\mathrm{~N}_{2}\left(C^{3} \Pi_{u}\right) \rightarrow \mathrm{N}_{2}\left(B^{3} \Pi_{g}\right)$ & $\sim 11$ & $50 \mathrm{~ns}$ & $\sim 30$ \\
$\mathrm{LBH} \mathrm{N}_{2}$ & $\mathrm{~N}_{2}\left(a^{1} \Pi_{u}\right) \rightarrow \mathrm{N}_{2}\left(X^{1} \Sigma_{g}^{+}\right)$ & $\sim 8.55$ & $14 \mu \mathrm{s}$ & $\sim 77$ \\
$1 \mathrm{NN}_{2}^{+}$ & $\mathrm{N}_{2}^{+}\left(B^{2} \Sigma_{u}^{+}\right) \rightarrow \mathrm{N}_{2}^{+}\left(X^{2} \Sigma_{g}^{+}\right)$ & $\sim 18.8$ & $69 \mathrm{~ns}$ & $\sim 48$ \\
\hline
\end{tabular}

The collisional excitation rates needed to obtain the fluorescence efficiencies are derived from kinetic calculations of the electron distribution function; the relevant states are noted in Table 1.

In this section we provide a review of related observations. Spectra of sprites in the stratosphere/mesosphere above electrically active cumulonimbus clouds were first acquired by Hampton et al. (1996) on 22 June, 1995, from an observation site atop Mt. Evens, and independently by Mende et al. (1995) on 16 July 1995, from an observation site near Fort Collins, Colorado. The Hampton et al. (1996) observations used a video slit spectrograph to obtain optical spectra of sprites. Twenty-five events were observed in the wavelength range $540-840 \mathrm{~nm}$ with spectral resolution approximately $6 \mathrm{~nm}$ and recorded with a $33 \mathrm{~ms}$ time resolution. The Mende et al. (1995) observations were conducted with a spectral resolution of approximately $9 \mathrm{~nm}$ and spectra recorded at a normal video rate (33 ms/frame) using an imaging spectrometer covering the wavelength range $\sim 450-800 \mathrm{~nm}$. Both sets of observations detected four distinct features in the 600-760 $\mathrm{nm}$ region which were identified as the $\mathrm{N}_{2}$ First Positive system with $\Delta=2,3$ and 4 from the $v=2,4,5,6$ vibrational levels of the $\mathrm{N}_{2}\left(B^{3} \Pi_{g}\right)$ state.

The spectral observations of both groups were analyzed in detail by Green et al. (1996) using energy dependent electron excitation cross sections and laboratory data to extract information on the vibrational distributions of the excited $\mathrm{N}_{2}\left(B^{3} \Pi_{g}\right)$ state and the energies of electrons producing the red sprite radiance. It was concluded that the sprite electrons appear to be of energy sufficient to dissociate and ionize $\mathrm{N}_{2}$. Results indicated excitation by electrons with a Boltzmann temperature of $1 \mathrm{eV}$ (range 0.4-2 eV). Green et al. (1996) also derived an estimate for the electric field magnitude driving sprite phenomenon of 100$200 \mathrm{~V} \mathrm{~m}^{-1}$ at $70 \mathrm{~km}$ altitude. This field appears to be fully consistent with the breakdown field.

The spectral resolution (6-9 nm) employed in Mende et al. (1995), Hampton et al. (1996) has not permitted accurate rotational temperature determination (Green et al. 1996), and we note that up to the present date there are no data available on the rotational temperature in sprites. In existing sprite models the rotational lines are computed at a temperature 220-230 K (Green et al. 1996; Bucsela et al. 2003). Recently, well-distinguishable infrasound signatures of sprite events have been reported (Liszka 2004; Farges et al. 2005; Liszka and Hobara 2006), indicating a possibility of heating of ambient atmospheric gas by sprite discharges. The measurements of rotational intensity distributions of $\mathrm{N}_{2}$ molecular bands may be potentially used for remote sensing of variations of gas temperature in sprite discharges (Phillips 1976; Vallance-Jones 1974, p. 157).

A time-dependent $\mathrm{N}_{2}$ vibrational level population model has been used in (Morrill et al. 1998) to simulate the spectral distributions and absolute intensities observed in sprites. Comparison of modeling results with the sprite spectrum taken at the TV field rate (17 ms resolution) measured with 7-11 nm resolution from the Wyoming Infrared Observatory (WORO) 
on Jelm Mountain during July 1996 led to a vibrational distribution of the $\mathrm{N}_{2}\left(B^{3} \Pi_{g}\right)$, which required an average electron energy of only $1-2 \mathrm{eV}$, generally consistent with earlier results reported in (Green et al. 1996). Analysis also indicated the presence of weak spectral features that were attributed to $\mathrm{N}_{2}^{+}$Meinel emissions (Morrill et al. 1998). Additional analysis of a sprite spectrum from $53 \mathrm{~km}$ altitude from the same data set has been conducted in Bucsela et al. (2003). The obtained $\mathrm{N}_{2}\left(B^{3} \Pi_{g}\right)$ vibrational distribution appeared to be consistent with those observed in laboratory afterglows, indicating an energy transfer process at lower altitudes in sprites (i.e. in sprite tendrils) between vibrationally excited $\mathrm{N}_{2}$ ground state and the lowest-energy, metastable electronic state:

$$
\begin{aligned}
& \mathrm{N}_{2}\left(A^{3} \Sigma_{u}^{+}, w\right)+\mathrm{N}_{2}\left(X^{1} \Sigma_{g}^{+}, v \geq 5\right) \\
& \quad \rightarrow \mathrm{N}_{2}\left(B^{3} \Pi_{g}, w^{\prime}\right)+\mathrm{N}_{2}\left(X^{1} \Sigma_{g}^{+}, v^{\prime} \sim 0\right) .
\end{aligned}
$$

The recently reported altitude-resolved sprite spectra (Kanmae et al. 2007) recorded with an imaging spectrograph with $3 \mathrm{~ms}$ and $3 \mathrm{~nm}$ temporal and spectral resolution, respectively, are consistent with this hypothesis. The metastable oxygen molecules $\mathrm{O}_{2}\left(a^{1} \Delta_{g}\right)$ are abundantly produced in streamer discharges (Lowke 1992; Naidis 1999), and a possible contribution to sprite $\mathrm{N}_{2}\left(B^{3} \Pi_{g}\right)$ emissions of energy transfer between $\mathrm{O}_{2}\left(a^{1} \Delta_{g}\right)$ and $\mathrm{N}_{2}\left(A^{3} \Sigma_{u}^{+}\right)$ metastable species has recently been discussed in Kamaratos (2006). However, in a simulation of the plasma chemistry associated with sprite streamers at $70 \mathrm{~km}$ altitude Sentman et al. (2008) found that this process is not a major contributor to sprite optical emissions.

The low temporal resolution $(<17-33 \mathrm{~ms})$ of spectral measurements in Mende et al. (1995), Hampton et al. (1996), and in the spectra used for subsequent analysis reported in Green et al. (1996), Bucsela et al. (2003), Morrill et al. (1998), represents a likely reason why more energetic electrons and higher electric fields, associated with streamer tips, have not been detected in these early measurements. A sub-millisecond time resolution is needed for accurate studies of sprite streamers. Following original observations of Mende et al. (1995), Hampton et al. (1996), narrow band photometric and blue-light video observations of sprites had been conducted (Armstrong et al. 1998, 2000; Suszcynsky et al. 1996), which indicated presence of a short $(<1 \mathrm{~ms})$ energetic ionizing event at the initial stage of sprite formation sufficient to ionize and excite molecular nitrogen, followed by secondary lower energy processes which give rise to the dominant and relatively long-lasting red emission. Specifically, sub-millisecond time resolution data on the $399.8 \mathrm{~nm} \mathrm{~N}_{2}(1,4)$ Second Positive band, $427.8 \mathrm{~nm} \mathrm{~N}_{2}^{+}(0,1)$ and $470.9 \mathrm{~N}_{2}^{+}(0,2)$ First Negative bands generated by sprites were analyzed in conjunction with supporting video imaging in Armstrong et al. (1998, 2000), Suszcynsky et al. (1996). The measured impulsive ionization emission during the sprite initiation exhibited an exponential decay time constant of only $0.3 \mathrm{~ms}$. The presence of more energetic electrons at the initial stage of sprite formation has also been confirmed by subsequent photometric observations reported in Miyasato et al. (2003), Takahashi et al. (2000).

During the EXL98 aircraft mission, sprites were observed by narrow band cameras that measure the $\mathrm{N}_{2}^{+}$First Negative $(0,1)$ band at $427.8 \mathrm{~nm}$ and the $\mathrm{N}_{2}$ Second Positive $(0,0)$ band at $337.0 \mathrm{~nm}$ (Morrill et al. 2002). The observations integrated the sprite emissions over $33 \mathrm{~ms}$ so that temporal information was limited. The analysis indicated characteristic electron energies on the order of $2 \mathrm{eV}$ and electric field magnitudes which closely followed the breakdown threshold field up to $55 \mathrm{~km}$ altitude and dropped below that level above $55 \mathrm{~km}$ (Morrill et al. 2002). These results are generally consistent with previous observations conducted with similar time resolution. 
The recently launched FORMOSAT-2 satellite carries the Imager for Sprites and Upper Atmospheric Lightning (ISUAL) instrument (Chern et al. 2003; Mende et al. 2005, 2006; Frey et al. 2005). The ISUAL science payload provides a unique opportunity to conduct a global survey of sprites and other TLEs from space using an intensified CCD imager, a six channel spectrophotometer and two array photometers (Mende et al. 2006), avoiding many complications associated with observations from ground-based and airborne platforms due to atmospheric transmission and absorption effects in the blue, violet and ultraviolet regions of the spectrum. Recently, in addition to the high time resolution photometric data on $1 \mathrm{PN}_{2}$, $2 \mathrm{PN}_{2}$ and $1 \mathrm{NN}_{2}^{+}$sprite emissions, the ISUAL instrument has successfully observed far-UV (FUV) emissions from sprites due to the $\mathrm{N}_{2}$ Lyman-Birge-Hopfield (LBH) band system (Mende et al. 2005, 2006; Frey et al. 2005).

\section{Implications for Planetary Atmospheres}

The kinetic theory and computational results presented in this introductory section form the basis for understanding the initiation and development of discharges in the gas mixtures that comprise planetary and lunar atmospheres. Both conventional breakdown and the recently discovered runaway mechanism were addressed and detailed solutions of the non-relativistic and relativistic Boltzmann equations presented. Our preliminary findings lead us to the following relevant conclusions:

- The threshold electric field for runaway breakdown is $\sim 6.3$ times lower on the gas giants, about equal on the moons, and $\sim 1.5$ times larger on Venus and Mars.

- For the same electric field and atmospheric density, the runaway avalanche time is (a) smaller on the gas giants by large factors near the runaway threshold on Jupiter and by a factor of $\sim 2$ to 3 at the higher electric fields, (b) larger on Mars and Venus by a factor of $\sim 1.3$, and (c) approximately equal on the moons when compared to the equivalent avalanche time on Earth.

- The Bremsstrahlung spectrum expected from a runaway discharge would be softer on the gas giants, slightly softer on the moons, and harder on Mars and Venus for the same electric field and atmospheric density.

- Emissions (RF and $\gamma$-ray) from the runaway beam would be highly collimated on the gas giants.

- The threshold electric field for conventional breakdown is a factor of $\sim 2$ times lower for the gas giants, $\sim 1.3$ time larger for Mars and Venus, and about the same for the moons compared to Earth.

The precise manner in which an electrical discharge would evolve on a given planet depends on the magnitude and atmospheric profile of the electric fields. In other words, the charging mechanisms and the gas density profile are crucial to establishing the conditions that are conducive to gas breakdown. These issues are addressed in other parts of this manuscript. We can however indicate, in agreement with Dwyer et al. (2006), that under similar conditions runaway breakdown is more likely to occur on the gas giants than conventional breakdown when compared to Earth or the other planets.

With these results in mind it is relevant to ask what we might expect to see on the other planets in light of what we observe on Earth given that our present understanding is that both conventional breakdown and runaway breakdown are at work. Of the many forms that a discharge can take in the terrestrial environment, lightning is by far the most spectacular and the most dangerous. The amount of energy expended in a single event is generally 
more than gigajoules with power levels reaching tens to hundreds of gigawatts. The currents that flow in a cloud-to-ground (CG) discharge range in magnitude from hundreds of amps to hundreds of kiloamps and transfer Coulombs to tens of Coulombs of charge. The bulk of this electrical energy flows through small cross-sectional areas with radii ranging from centimeters to tens of centimeters and over long distances extending to many kilometers. The kinetic energy density in lightning is sufficient in many cases to heat the air to tens of thousands of degrees Kelvin and to generate acoustic shock waves that can be heard out to tens of kilometers. Terrestrial lightning is easily observed from the ground and from space in the optical, the radio frequency, and most recently in the X-ray and gamma ray parts of the electromagnetic spectrum. The physical manifestations of the lightning discharge are so diverse as to precipitate the proliferation of a descriptive and non-descriptive taxonomy that includes ribbons, balls, jets, sprites, elves, halos, angels, starters, trolls, beads, carrots, superbolts, and spiders.

The temptation to extrapolate the properties of terrestrial lightning to other planets and astrophysical systems is great. However, our present understanding of this seemingly well understood and extensively studied physical process is undergoing a significant change as a result of the many recent measurements of penetrating radiation in coincidence with stepped leader processes, intra-cloud discharges, and potentially high-altitude discharges. Early attempts to rely on laboratory discharge experiments to understand the lightning phenomenon met with some success but ultimately could not explain the almost ubiquitous presence of energetic radiation. Our final understanding of this incredibly fascinating natural phenomenon will not rest on a phenomenological taxonomy nor on extrapolation of small-scale laboratory experiments but rather on a fundamental shift in our thinking that must ultimately include a role for cosmic rays, relativistic electron beams, large scales, and energetic feedback processes. The detection of lightning on other planets by means of diagnostics that span the electromagnetic spectrum will help us understand the fundamental nature of the discharge process.

Acknowledgements The participation of V.P. Pasko has been supported by the United States National Science Foundation under the NSF ATM-0741589 grant to Penn State University.

\section{References}

F.L. Addessio, J.R. Baumgardner, J.K. Dukowicz et al., Caveat: A computer code for fluid dynamics problems with large distortion and internal slip, 1992

R.A. Armstrong, J.A. Shorter, M.J. Taylor et al., J. Atmos. Sol. Terr. Phys. 60, 787 (1998)

R.A. Armstrong, D.M. Suszcynsky, W.A. Lyons et al., Geophys. Res. Lett. 27, 653 (2000)

T. Asano, M. Hayakawa, M. Cho et al., J. Geophys. Res.-Space Phys. 113, A02308 (2008)

L.P. Babich, E.N. Donskoy, I.M. Kutsyk et al., IEEE Trans. Plasma Sci. 29, 430 (2001)

L.P. Babich, E.N. Donskoy, K.F. Zelenskii et al., Dokl. Phys. 47, 1 (2002)

L.P. Babich, A.Y. Kudryavtsev, M.L. Kudryavtseva et al., J. Exp. Theor. Phys. 106, 65 (2008)

C.P. Barrington-Leigh, U.S. Inan, M. Stanley, J. Goephys. Res. 101, 1741 (2001)

E.M. Bazelyan, Y.P. Raizer, Spark Discharge (CRC Press, Boca Raton, 1998)

H.A. Bethe, Ann. Phys. 5, 325 (1930)

H.A. Bethe, J. Ashkin, in Experimental Nuclear Physics, ed. by E. Segre (Wiley, New York, 1953), p. 277

S.F. Biagi, Nucl. Inst. Methods A 421, 234 (1999)

E. Blanc, F. Lefeuvre, R. Roussel-Dupré et al., Adv. Space Res. 40, 1268 (2007)

D.J. Boccippio, E.R. Williams, S.J. Heckman et al., Science 269, 1088 (1995)

T.M.P. Briels, E.M. van Veldhuizen, U. Ebert, IEEE Trans. Plasma Sci. 33, 264 (2005)

M.J. Brunger, S.J. Buckman, L.J. Allen et al., J. Phys. B: At. Mol. Opt. Phys. 25, 1823 (1992)

M.J. Brunger, S.J. Buckman, Phys. Rep. 357, 215 (2002)

E. Bucsela, J. Morrill, M. Heavner et al., J. Atmos. Sol. Terr. Phys. 65, 583 (2003) 
S. Chapman, T.G. Cowling, The Mathematical Theory of Non-uniform Gases (Cambridge University Press, London, 1970)

B. Chen, Y. Lee, R. Hsu et al., Global distribution and seasonal distribution variation of transient luminous events. AGU Fall meeting, San Francisco, 2005

J.L. Chern, R.R. Hsu, H.T. Su et al., J. Atmos. Sol. Terr. Phys. 65, 647 (2003)

H.J. Christian, R.J. Blakeslee, D.J. Boccippio et al., J. Geophys. Res. 108 (2003)

S.A. Cummer, N. Jaugey, J. Li et al., Geophys. Res. Lett. 33, L04104 (2006a)

S.A. Cummer, H.U. Frey, S.B. Mende et al., J. Geophys. Res. 111, A10315 (2006b)

H. Dreicer, Phys. Rev. 115(2), 238 (1959)

H. Dreicer, Phys. Rev. 117(2), 343 (1960)

J.R. Dwyer, Geophys. Res. Lett. 30, 2055 (2003)

J.R. Dwyer, D.M. Smith, Geophys. Res. Lett. 32, L22804 (2005)

J.R. Dwyer, L.M. Coleman, R. Lopez et al., Geophys. Res. Lett. 33, L22813 (2006)

J.R. Dwyer, B.W. Grefenstette, D.M. Smith, Geophys, Res. Lett. 35, L02815 (2008)

K.B. Eack, W.B. Beasley, W.D. Rust et al., Geophys. Res. Lett. 23, 2915 (1996)

U. Ebert, C. Montijn, T.M.P. Briels et al., Plasma Sources Sci. Technol. 15, S118 (2006)

C.F. Enell, E. Arnone, T. Adachi et al., Ann. Geophys. 26, 12 (2008)

T. Farges, E. Blanc, A. Le Pichon et al., Geophys. Res. Lett. 32, L01813 (2005)

G.J. Fishman, P.N. Bhat, R. Mallozzi et al., Science 264, 1313 (1994)

H.U. Frey, S.B. Mende, S.A. Cummer et al., Geophys. Res. Lett. 32, L13824 (2005)

H. Fukunishi, Y. Takahashi, M. Kubota et al., Geophys. Res. Lett. 23, 2157 (1996)

E.A. Gerken, U.S. Inan, J. Geophys. Res 107, 1344 (2002)

E.A. Gerken, U.S. Inan, J. Atmos. Sol. Terr. Phys. 65, 567 (2003)

E.A. Gerken, U.S. Inan, IEEE Trans. Plasma Sci. 33, 282 (2005)

E.A. Gerken, U.S. Inan, C.P. Barrington-Leigh, Geophys. Res. Lett. 27, 2637 (2000)

B.D. Green, M.E. Fraser, W.T. Rawlins et al., Geophys. Res. Lett. 23, 2161 (1996)

B.W. Grefenstette, D.M. Smith, J.R. Dwyer et al., Geophys. Res. Lett. 35, L06802 (2008)

A.V. Gurevich, K.P. Zybin Phys. Today 37 (2005)

A.V. Gurevich, G.M. Milikh, R.A. Roussel-Dupré, Phys. Lett. A 165, 463 (1992)

A.V. Gurevich, G.M. Milikh, R.A. Roussel-Dupré, Phys. Lett. A 187, 197 (1994)

A.V. Gurevich, J.A. Valdivia, G.M. Milikh et al., Radio Sci. 31, 1541 (1996)

A.V. Gurevich, R.A. Roussel-Dupré, K.P. Zybin, Phys. Lett. A 237, 240 (1998)

A.V. Gurevich, K.F. Sergeichev, I.A. Sychov et al., Phys. Lett. A 260, 269 (1999)

D.L. Hampton, M.J. Heavner, E.M. Wescott et al., Geophys. Res. Lett. 23 (1996)

M.J. Heavner, D.D. Sentman, D.R. Moudry et al., in Geophysical Monograph Series, ed. by D.E. Siskind, S.D. Eckerman, M.E. Summers (Am. Geophys. Union, Washington, 2000)

L.G.H. Huxley, R.W. Crompton, The Diffusion and Drift of Electrons in Gases (Wiley, New York, 1974)

ICRU, ICRU Report No. 49, ed. by Bethesda, 1993

U.S. Inan, C. Barrington-Leigh, S. Hansen et al., Geophys. Res. Lett. 24, 583 (1997)

Y. Itikawa, J. Phys. Chem. Ref. Data 31, 3 (2002)

Y. Itikawa, J. Phys. Chem. Ref. Data 35, 31 (2006)

B.-H. Jeon, J. Korean Phys. Soc. 43, 513 (2003)

B.-H. Jeon, Y. Nakamura, J. Phys. D 31, 2145 (1998)

E. Kamaratos, Chem. Phys. 323, 271 (2006)

I. Kanik, S. Trajmar, J.C. Nickel, J. Geophys. Res. 98, 7447 (1993)

T. Kanmae, H.C. Stenbaek-Nielsen, M.G. McHarg, Geophys. Res. Lett. 34, L07810 (2007)

N.A. Krall, A.W. Trivelpiece, Principles of Plasma Physics (McGraw-Hill, New York, 1973)

C.-L. Kuo, A.B. Chen, Y.J. Lee et al., J. Geophys. Res. 112, A11312 (2007)

N.G. Lehtinen, T.F. Bell, U.S. Inan, J. Geophys. Res. 104, 24 (1999)

L. Liszka, J. Low Freq. Noise, Vib. Act. Control 23, 85 (2004)

L. Liszka, Y. Hobara, J. Atmos. Sol.-Terr. Phys. 68, 1179 (2006)

N. Liu, V.P. Pasko, J. Geophys. Res.-Space Phys. 109, A04301 (2004)

N. Liu, V.P. Pasko, J. Phys. D: Appl. Phys. 39, 327 (2006)

N. Liu, V.P. Pasko, D.H. Burkhardt et al., Geophys. Res. Lett. 33, L01101 (2006)

L.B. Loeb, Fundamental Processes of Electrical Discharge in Gases (Wiley, New York, 1939)

J.J. Lowke, J. Phys. D: Appl. Phys. 25, 202 (1992)

W.A. Lyons, J. Geophys. Res. 101, 29641 (1996)

W.A. Lyons, in Sprites, Elves and Intense Lightning Discharges, ed. by M. Füllekrug, E.A. Mareev, M.J. Rycroft (Springer, Berlin, 2006)

W.A. Lyons, T.E. Nelson, R.A. Armstrong et al., Am. Meteorol. Soc. 445 (2003a)

W.A. Lyons, T.E. Nelson E, R. Williams et al., Mon. Weather Rev. 131, 2417 (2003b) 
R.A. Marshall, U.S. Inan, Geophys. Res. Lett. 32, L05804 (2005)

R.A. Marshall, U.S. Inan, Radio Sci. 41, RS6S43 (2006)

T.C. Marshall, M. Stolzenburg, C.R. Maggio et al., Geophys. Res. Lett. 32, L03813 (2005)

M.P. McCarthy, G.K. Parks, Geophys. Res. Lett. 12, 393 (1985)

M.P. McCarthy, G.K. Parks, J. Geophys. Res. Lett. 97, 5857 (1992)

S.B. Mende, R.L. Rairden, G.R. Swenson et al., Geophys. Res. Lett. 22, 2633 (1995)

S.B. Mende, H.U. Frey, R.R. Hsu et al., J. Geophys. Res. 110, A11312 (2005)

S.B. Mende, Y.S. Chang, A.B. Chen et al., in Sprites, Elves and Intense Lightning Discharges, ed. by M. Fullekrug, E.A. Mareev, M.J. Rycroft (Springer, Berlin, 2006)

R. Miyasato, H. Fukunishi, Y. Takahashi et al., J. Atmos. Sol. Terr. Phys. 65, 573 (2003)

C.B. Moore, K.B. Eack, G.D. Aulich et al., Geophys. Res. Lett. 28, 2141 (2001)

J.S. Morrill, E.J. Bucsela, V.P. Pasko et al., J. Atmos. Sol. Terr. Phys. 60, 811 (1998)

J. Morrill, E. Bucsela, C. Seifring et al., Geophys. Res. Lett. 29, 1462 (2002)

G.D. Moss, V.P. Pasko, N. Liu et al., J. Geophys. Res. 111, A02307 (2006)

G.V. Naidis, J. Phys. D: Appl. Phys. 32, 2649 (1999)

N. Østgaard, T. Gjesteland, J. Stadsnes et al., J. Geophys. Res. 113, A02307 (2008)

S.V. Pancheshnyi, M. Nudnova, A.Y. Starikovskii, Phys. Rev. E 71, 016407 (2005)

V.P. Pasko, Nature 423, 927 (2003)

V.P. Pasko, in Sprites, Elves and Intense Lightning Discharges, ed. by M. Füllekrug, E.A. Mareev, M.J. Rycroft (Springer, Berlin, 2006)

V.P. Pasko, U.S. Inan, T.F. Bell, Geophys. Res. Lett. 25, 2123 (1998)

V.P. Pasko, M.A. Stanley, J.D. Mathews et al., Nature 416, 152 (2002)

V.P. Pasko, H.C. Stenbaek-Nielsen, Geophys. Res. Lett. 29, 1440 (2002)

A.V. Phelps, L.C. Pitchford, Technical Report \#26, JILA Information Center Report, University of Colorado, Boulder, CO, USA (1985)

D.M. Phillips, J. Phys. D: Appl. Phys. 9, 507 (1976)

L.C. Pitchford, A.V. Phelps, Phys. Rev. A 25, 540 (1982)

H. Raether, Electron Avalanches and Breakdown in Gase (Butterworths, London, 1964)

Y.P. Raizer, Gas Discharge Physics (Springer, New York, 1991)

R.A. Roussel-Dupré, A.V. Gurevich, J. Geophys. Res. 101, 2297 (1996)

R.A. Roussel-Dupré, A.V. Gurevich, T. Tunnell et al., Phys. Rev. E 49, 2257 (1994)

R.A. Roussel-Dupré, E.M.D. Symbalisty, L. Triplett et al., Cal Meeting, Crete, 2005

M.B. Scott, A.O. Hanson, E.M. Lyman, Phys. Rev. 84, 638 (1951)

D.D. Sentman, E.M. Wescott, Geophys. Res. Lett. 22, 1205 (1993)

D.D. Sentman, E.M. Wescott, D.L. Osborne et al., Geophys. Res. Lett. 22, 1205 (1995)

D.D. Sentman, H.C. Stenbaek-Nielsen, M.G. McHarg et al., J. Geophys. Res.: Atmos. (2008, in press)

D.M. Smith, L.I. Lopez, R.P. Lin et al., Science 307, 1085 (2005)

M. Stanley, P. Krehbiel, M. Brook et al., Geophys. Res. Lett. 26, 3201 (1999)

H.C. Stenbaek-Nielsen, D.R. Moudry, E.M. Wescott et al., Geophys. Res. Lett. 27, 3829 (2000)

M. Stolzenburg, T.C. Marshall, W.D. Rust et al., Geophys. Res. Lett. 34, L04804 (2007)

H.T. Su, R.R. Hsu, A.B. Chen et al., Nature 423, 974 (2003)

D.M. Suszcynsky, R. Roussel-Dupré, G. Shaw, J. Geophys. Res. 101, 23 (1996)

E.M.D. Symbalisty, R. Roussel-Dupré, V. Yukhimuk, IEEE Trans. Plasma Sci. 26, 1575 (1998)

Y. Takahashi, M. Fujito, Y. Watanabe et al., Adv. Space Res. 26, 1205 (2000)

P. Tardiveau, E. Marode, J. Phys. D: Appl. Phys. 36 (2003)

P. Tardiveau, E. Marode, A. Agneray et al., J. Phys. D: Appl. Phys. 34, 1690 (2001)

H. Tawara, Y. Itikawa, H. Nishimura et al., J. Phys. Chem. Ref. Data 19, 617 (1990a)

H. Tawara, Y. Itikawa, H. Nishimura et al., NIFS-DATA-6, 1990b

S. Trajmar, D.F. Register, A. Chutjian, Phys. Rep. 97, 219 (1983)

M.A. Uman, The Lightning Discharge (Dover, New York, 2001)

A.V. Vallance-Jones, Aurora (Reidel, Norwell, 1974)

E.M. van Veldhuizen, Electrical Discharges for Environmental Purposes: Fundamentals and Applications (Nova Science, New York, 2000)

E.M. Wescott, D. Sentman, D. Osborne et al., Geophys. Res. Lett. 22, 1209 (1995)

C.T.R. Wilson, Proc. R. Soc. Lond. 37, 32D (1925)

C.T.R. Wilson, Proc. R. Soc. Lond. 236, 297 (1956) 